\title{
Toxicity Profiling of Engineered Nanomaterials via Multivariate Dose Response Surface Modeling
}

\author{
TRINA PATEL ${ }^{1}$, DONATELLO TELESCA ${ }^{1}$, \\ SAJI GEORGE ${ }^{2,3}$, ANDRÉ E. NEL ${ }^{2,3}$ \\ Author's Footnote \\ ${ }^{1}$ UCLA School of Public Health, Department of Biostatistics. \\ ${ }^{2}$ UCLA Department of Medicine, Division of NanoMedicine. \\ ${ }^{3}$ UCLA California NanoSystems Institute.
}

July 22, 2012

\section{SUPPLEMENTARY MATERIAL}

\section{Appendix A: Full Conditional Distributions}

In this appendix we describe some of the full conditional distributions for the model described in the paper. Let $y_{i j k}(d, t)$ denote a multivariate response corresponding to $\mathrm{ENM} i(i=$ $1, \ldots, I)$, cytotoxicity parameter $j(j=1, \ldots, J)$, and replicate $k(k=1, \ldots, K)$ at some dose $d$ in $d=\left(d_{1}, \ldots d_{D}\right)^{\prime}$ and some time $t$ in $\boldsymbol{t}=\left(t_{1}, \ldots t_{T}\right)^{\prime}$.

Also, let $\boldsymbol{\theta}=\left(\boldsymbol{\alpha}, \boldsymbol{\beta}, \boldsymbol{\gamma}, \boldsymbol{\phi}, \boldsymbol{\psi}, \boldsymbol{\alpha}_{\boldsymbol{o}}, \boldsymbol{\beta}_{\boldsymbol{o}}, \boldsymbol{\gamma}_{\boldsymbol{o}}, \boldsymbol{\tau}, \boldsymbol{\sigma}_{\boldsymbol{\epsilon}}, \boldsymbol{\sigma}_{\boldsymbol{\alpha}}, \boldsymbol{\sigma}_{\boldsymbol{\beta}}, \boldsymbol{\sigma}_{\gamma}\right)$ denote the full parameter vector and, let $\boldsymbol{\theta} \backslash \boldsymbol{\delta}$ denote the vector containing all components of $\boldsymbol{\theta}$ except for some parameter $\delta$ in $\boldsymbol{\theta}$. Moreover, we denote $\boldsymbol{Y}_{\boldsymbol{i} \boldsymbol{j}}$ as the complete set of response values for particle $i$ and outcome $j$. Using the notation above we define the full conditional distributions for all available parameters as follows.

A.1.1: Full conditional distributions for the random effect parameters $\alpha_{i j}, \boldsymbol{\beta}_{\boldsymbol{i j}}, \boldsymbol{\gamma}_{\boldsymbol{i} \boldsymbol{j}}$, and $\boldsymbol{\delta}_{\boldsymbol{i} \boldsymbol{j}}$. 
$\alpha_{i j} \mid \boldsymbol{Y}_{\boldsymbol{i j}}, \boldsymbol{\theta}_{\backslash \alpha_{i j}} \sim N\left(\frac{\frac{\sum_{d, t, k} \tilde{y}_{\alpha_{i j k}}(d, t)}{\sigma_{\epsilon_{j}}^{2} / \tau_{i}}+\frac{\alpha_{o_{i}}}{\sigma \alpha_{i}}}{\frac{D \times T \times K}{\sigma_{\epsilon_{j}}^{2} / \tau_{i}}+\frac{1}{\sigma \alpha_{i}}}, \frac{1}{\frac{D \times T \times K}{\sigma_{\epsilon_{j}}^{2} / \tau_{i}}+\frac{1}{\sigma \alpha_{i}}}\right)$,

where

$\tilde{y}_{\alpha_{i j k}}(d, t)= \begin{cases}y_{i j k}(d, t)-\mathcal{B}\left(d, \boldsymbol{\phi}_{i j}\right)^{\prime} \boldsymbol{\beta}_{i j}-\mathcal{B}\left(t, \boldsymbol{\psi}_{i j}\right)^{\prime} \boldsymbol{\gamma}_{i j} & \text { if } \rho_{i j}=0 \\ y_{i j k}(d, t)-\mathcal{B}\left(d, \boldsymbol{\phi}_{i j}\right)^{\prime} \boldsymbol{\beta}_{i j}-\mathcal{B}\left(t, \boldsymbol{\psi}_{i j}\right)^{\prime} \boldsymbol{\gamma}_{i j}-\mathcal{B}\left(d * t, \boldsymbol{\chi}_{i j}\right)^{\prime} \boldsymbol{\delta}_{i j} & \text { if } \rho_{i j}=1 .\end{cases}$

$\boldsymbol{\beta}_{\boldsymbol{i j}} \mid \boldsymbol{Y}_{\boldsymbol{i j}}, \boldsymbol{\theta}_{\backslash \boldsymbol{\beta}_{\boldsymbol{i j}}} \sim N_{4}\left(M_{\beta}, V_{\beta}\right)$,

where

$M_{\beta}=\left(\boldsymbol{\Sigma}_{\boldsymbol{\beta}_{\boldsymbol{i}}}^{-1}+\sum_{d, t, k} \frac{\mathcal{B}\left(d, \boldsymbol{\phi}_{i j}\right) \mathcal{B}\left(d, \boldsymbol{\phi}_{i j}\right)^{\prime}}{\sigma_{\epsilon_{j}}^{2} / \tau_{i}}\right)^{-1}\left(\boldsymbol{\Sigma}_{\boldsymbol{\beta}_{\boldsymbol{i}}}^{-1} \boldsymbol{\beta}_{\boldsymbol{o}_{\boldsymbol{i}}}+\sum_{d, t, k} \frac{\mathcal{B}\left(d, \boldsymbol{\phi}_{i j}\right) \tilde{y}_{\beta_{i j k}}(d, t)}{\sigma_{\epsilon_{j}}^{2} / \tau_{i}}\right)$,
$V_{\beta}=\left(\boldsymbol{\Sigma}_{\boldsymbol{\beta}_{\boldsymbol{i}}}^{-1}+\sum_{d, t, k} \frac{\mathcal{B}\left(d, \boldsymbol{\phi}_{i j}\right) \mathcal{B}\left(d, \boldsymbol{\phi}_{i j}\right)^{\prime}}{\sigma_{\epsilon_{j}}^{2} / \tau_{i}}\right)^{-1}$

$\tilde{y}_{\beta_{i j k}}(d, t)= \begin{cases}y_{i j k}(d, t)-\mathcal{B}\left(t, \boldsymbol{\psi}_{i j}\right)^{\prime} \boldsymbol{\gamma}_{i j}-\alpha_{i j} & \text { if } \rho_{i j}=0 \\ y_{i j k}(d, t)-\mathcal{B}\left(t, \boldsymbol{\psi}_{i j}\right)^{\prime} \boldsymbol{\gamma}_{i j}-\mathcal{B}\left(d * t, \boldsymbol{\chi}_{i j}\right)^{\prime} \boldsymbol{\delta}_{i j}-\alpha_{i j} & \text { if } \rho_{i j}=1 .\end{cases}$

$\gamma_{i j} \mid \boldsymbol{Y}_{i j}, \boldsymbol{\theta}_{\backslash \gamma_{i j}} \sim N_{4}\left(M_{\gamma}, V_{\gamma}\right)$,

where

$M_{\gamma}=\left(\boldsymbol{\Sigma}_{\gamma_{i}}^{-1}+\sum_{d, t, k} \frac{\mathcal{B}\left(t, \boldsymbol{\psi}_{i j}\right) \mathcal{B}\left(t, \boldsymbol{\psi}_{i j}\right)^{\prime}}{\sigma_{\epsilon_{j}}^{2} / \tau_{i}}\right)^{-1}\left(\boldsymbol{\Sigma}_{\gamma_{i}}^{-1} \gamma_{\boldsymbol{o}_{\boldsymbol{i}}}+\sum_{d, t, k} \frac{\mathcal{B}\left(t, \boldsymbol{\psi}_{i j}\right) \tilde{y}_{\gamma_{i j k}(d, t)}}{\sigma_{\epsilon_{j}}^{2} / \tau_{i}}\right)$,

$V_{\gamma}=\left(\Sigma_{\gamma_{i}}^{-1}+\sum_{d, t, k} \frac{\mathcal{B}\left(t, \boldsymbol{\psi}_{i j}\right) \mathcal{B}\left(t, \boldsymbol{\psi}_{i j}\right)^{\prime}}{\sigma_{\epsilon_{j}}^{2} / \tau_{i}}\right)^{-1}$

$\tilde{y}_{\gamma_{i j k}}(d, t)= \begin{cases}y_{i j k}(d, t)-\mathcal{B}\left(d, \boldsymbol{\phi}_{i j}\right)^{\prime} \boldsymbol{\beta}_{i j}-\alpha_{i j} & \text { if } \rho_{i j}=0 \\ y_{i j k}(d, t)-\mathcal{B}\left(d, \boldsymbol{\phi}_{i j}\right)^{\prime} \boldsymbol{\beta}_{i j}-\mathcal{B}\left(d * t, \boldsymbol{\chi}_{i j}\right)^{\prime} \boldsymbol{\delta}_{i j}-\alpha_{i j} & \text { if } \rho_{i j}=1 .\end{cases}$

$\boldsymbol{\delta}_{\boldsymbol{i j}} \mid \boldsymbol{Y}_{\boldsymbol{i j}}, \boldsymbol{\theta}_{\backslash \boldsymbol{\delta}_{\boldsymbol{i j}}} \sim N_{4}\left(M_{\delta}, V_{\delta}\right)$,

where 


$$
\begin{aligned}
& M_{\delta}=\left(\boldsymbol{\Sigma}_{\boldsymbol{\delta}_{\boldsymbol{i}}}^{-1}+\sum_{d, t, k} \frac{\mathcal{B}\left(t, \boldsymbol{\chi}_{i j}\right) \mathcal{B}\left(d * t, \boldsymbol{\chi}_{i j}\right)^{\prime}}{\sigma_{\epsilon_{j}}^{2} / \tau_{i}}\right)^{-1}\left(\boldsymbol{\Sigma}_{\boldsymbol{\delta}_{\boldsymbol{i}}}^{-1} \boldsymbol{m}_{\boldsymbol{\delta}_{\boldsymbol{i}}}+\sum_{d, t, k} \frac{\mathcal{B}\left(d * t, \boldsymbol{\chi}_{i j}\right) \tilde{y}_{\delta_{i j k}}(d, t)}{\sigma_{\epsilon_{j}}^{2} / \tau_{i}}\right), \\
& V_{\delta}=\left(\boldsymbol{\Sigma}_{\boldsymbol{\gamma}_{i}}^{-1}+\sum_{d, t, k} \frac{\mathcal{B}\left(t, \boldsymbol{\psi}_{i j}\right) \mathcal{B}\left(t, \boldsymbol{\psi}_{i j}\right)^{\prime}}{\sigma_{\epsilon_{j}}^{2} / \tau_{i}}\right)^{-1}, \\
& \tilde{y}_{\delta_{i j k}}(d, t)=y_{i j k}(d, t)-\mathcal{B}\left(d, \boldsymbol{\phi}_{i j}\right)^{\prime} \boldsymbol{\beta}_{i j}-\mathcal{B}\left(t, \boldsymbol{\psi}_{i j}\right)^{\prime} \boldsymbol{\gamma}_{i j}-\alpha_{i j} .
\end{aligned}
$$


A.1.2: Full conditional distributions for ENM level parameters $\alpha_{o_{i}}, \beta_{o_{i}}$, and $\gamma_{o_{i}}$.

$$
\begin{aligned}
& \alpha_{o_{i}} \mid \boldsymbol{\theta}_{\backslash \alpha_{i j}} \sim N\left(\frac{\frac{\sum_{j=1}^{J} \alpha_{i j}}{\sigma_{\alpha_{i}}^{2}}+\frac{m_{\alpha_{i}}}{s_{\alpha_{i}}^{2}}}{\frac{J}{\sigma_{\alpha_{i}}^{2}}+\frac{1}{s_{\alpha_{i}}^{2}}}, \frac{1}{\frac{J}{\sigma_{\alpha_{i}}^{2}}+\frac{1}{s_{\alpha_{i}}^{2}}}\right), \\
& \boldsymbol{\beta}_{\boldsymbol{o}_{\boldsymbol{i}}} \mid \boldsymbol{\theta}_{\backslash \boldsymbol{\beta}_{\boldsymbol{i}}} \sim N_{4}\left(\left(J \Sigma_{\boldsymbol{\beta}_{\boldsymbol{i}}}^{-1}+\boldsymbol{v}_{\boldsymbol{\beta}_{\boldsymbol{i}}}^{-1}\right)^{-1}\left(\boldsymbol{\Sigma}_{\boldsymbol{\beta}_{\boldsymbol{i}}}^{-1} \sum_{j=1}^{J} \boldsymbol{\beta}_{\boldsymbol{i}}+\boldsymbol{v}_{\boldsymbol{\beta}_{\boldsymbol{i}}}^{-1} \boldsymbol{m}_{\boldsymbol{\beta}_{\boldsymbol{i}}}\right),\left(J \Sigma_{\boldsymbol{\beta}_{\boldsymbol{i}}}^{-1}+\boldsymbol{v}_{\boldsymbol{\beta}_{\boldsymbol{i}}}^{-1}\right)^{-1}\right), \\
& \gamma_{o_{i}} \mid \boldsymbol{\theta}_{\backslash \gamma_{i j}} \sim N_{4}\left(\left(J \Sigma_{\gamma_{i}}^{-1}+\boldsymbol{v}_{\gamma_{i}}^{-1}\right)^{-1}\left(\Sigma_{\gamma_{i}}^{-1} \sum_{j=1}^{J} \gamma_{i j}+\boldsymbol{v}_{\gamma_{i}}^{-1} \boldsymbol{m}_{\gamma_{i}}\right),\left(J \Sigma_{\gamma_{i}}^{-1}+\boldsymbol{v}_{\gamma_{i}}^{-1}\right)^{-1}\right) \text {. }
\end{aligned}
$$

A.1.3: Full conditional distributions for the error variance parameter $\sigma_{\epsilon_{j}}^{2}$ and variance inflation parameter $\tau_{i}$.

$$
1 / \sigma_{\epsilon_{j}}^{2} \mid \boldsymbol{Y}_{i j}, \boldsymbol{\theta}_{\sigma_{\sigma_{j}}} \sim \operatorname{Gamma}\left(a_{\epsilon_{j}}+\frac{I \times D \times T \times K}{2}, \frac{1}{2} \sum_{d, t, k, i}\left(y_{i j k}(d, t)-m_{i j}(d, t)\right)^{2} \tau_{i}+b_{\epsilon_{j}}\right),
$$

where

$$
m_{i j}(d, t)= \begin{cases}\mathcal{B}\left(d, \boldsymbol{\phi}_{i j}\right)^{\prime} \boldsymbol{\beta}_{i j}+\mathcal{B}\left(t, \boldsymbol{\psi}_{i j}\right)^{\prime} \boldsymbol{\gamma}_{i j}+\alpha_{i j} & \text { if } \rho_{i j}=0 \\ \mathcal{B}\left(d, \boldsymbol{\phi}_{i j}\right)^{\prime} \boldsymbol{\beta}_{i j}+\mathcal{B}\left(t, \boldsymbol{\psi}_{i j}\right)^{\prime} \boldsymbol{\gamma}_{i j}+\mathcal{B}\left(d * t, \boldsymbol{\chi}_{i j}\right)^{\prime} \boldsymbol{\gamma}_{i j}+\alpha_{i j} & \text { if } \rho_{i j}=1 .\end{cases}
$$

$\tau_{i} \mid \boldsymbol{Y}_{i j}, \boldsymbol{\theta}_{\backslash \tau_{i}} \sim \operatorname{Gamma}\left(\frac{\nu}{2}+\frac{J \times K \times D \times T}{2}, \frac{1}{2} \sum_{d, t, k, j} \frac{\left(y_{i j k}(d, t)-m_{i j}(d, t)\right)^{2}}{\sigma_{\epsilon_{j}}^{2}}+\frac{\nu}{2}\right)$,

where

$$
m_{i j}(d, t)= \begin{cases}\mathcal{B}\left(d, \boldsymbol{\phi}_{i j}\right)^{\prime} \boldsymbol{\beta}_{i j}+\mathcal{B}\left(t, \boldsymbol{\psi}_{i j}\right)^{\prime} \boldsymbol{\gamma}_{i j}+\alpha_{i j} & \text { if } \rho_{i j}=0 \\ \mathcal{B}\left(d, \boldsymbol{\phi}_{i j}\right)^{\prime} \boldsymbol{\beta}_{i j}+\mathcal{B}\left(t, \boldsymbol{\psi}_{i j}\right)^{\prime} \boldsymbol{\gamma}_{i j}+\mathcal{B}\left(d * t, \boldsymbol{\chi}_{i j}\right)^{\prime} \boldsymbol{\gamma}_{i j}+\alpha_{i j} & \text { if } \rho_{i j}=1 .\end{cases}
$$

A.1.4: Full conditional distributions for other variance parameters

$$
\begin{aligned}
& 1 / \sigma_{\alpha_{i}}^{2} \mid \boldsymbol{\theta}_{\backslash \sigma_{\alpha_{i}}} \sim \operatorname{Gamma}\left(a_{\alpha i}+\frac{J}{2}, b_{\alpha_{i}}+\frac{1}{2} \sum_{j=1}^{J}\left(\alpha_{i j}-\alpha_{o_{i}}\right)^{2}\right), \\
& 1 / \sigma_{\beta_{i \ell}}^{2} \mid \boldsymbol{\theta}_{\sigma_{\sigma_{\beta_{i \ell}}}} \sim \operatorname{Gamma}\left(a_{\beta_{i}}+\frac{J}{2}, b_{\beta_{i}}+\frac{1}{2} \sum_{j=1}^{J}\left(\beta_{i j \ell}-\beta_{o_{i} \ell}\right)^{2}\right), \\
& 1 / \sigma_{\gamma_{i \ell}}^{2} \mid \boldsymbol{\theta}_{\backslash \sigma_{\gamma_{i \ell}}} \sim \operatorname{Gamma}\left(a_{\gamma_{i}}+\frac{J}{2}, b_{\gamma_{i}}+\frac{1}{2} \sum_{j=1}^{J}\left(\gamma_{i j \ell}-\gamma_{o_{i} \ell}\right)^{2}\right),
\end{aligned}
$$

where $\ell=1, . ., 4$. 


\section{Appendix B: Simulation Study Assessing Model Fit}

To assess estimation of the model presented in the paper, we simulated four sets, $(i=1, \ldots, 4)$, of four independent surfaces, $(j=1, \ldots, 4)$, each evaluated at ten doses $(d \in[0, D])$ and seven times of exposure $(t \in[0, T])$. The dose and time kinetics were simulated in an additive fashion from various parametric functions. In addition for each set of response-surfaces, we simulated a dose*time interaction function for two of the four surfaces. If we let $y_{i j k}(d, t)$ denote a multivariate response corresponding to a set of surfaces $i(i=1, \ldots, 4)$, outcome $j$

$(j=1, \ldots, 4)$, and replicate $k(k=1, \ldots, 4)$, at dose $d \in[0, D]$ and time $t \in[0, T]$, then each simulated surface can be described by

$$
y_{i j k}(d, t)=s_{i j}(d, t)+\xi_{i j k}(d, t) .
$$

Here $s_{i j}(d, t)=\alpha_{i j}+r_{i j}(d)+u_{i j}(t)+w_{i j}(d t) I\left(r_{i j}=1\right)$ represents a smooth dose-responsesurface and $r_{i j}$ an indicator that determines whether or not a dose-time interaction was simulated. The functions $r_{i j}(d), u_{i j}(t)$, and $w_{i j}(d t)$ were simulated from various parametric functions such as log-logistic, weibull, polynomial, and cubic spline models. These parametric functions were used to construct both canonical and non-canonical profiles that are still reasonably interpretable in a toxicity framework. Note that the simulated response curves do not follow our model which uses a B-spline representation with two random interior knots. Dose-response surfaces were also simulated with varying levels of noise, across each outcome $j$. This is as expected in high-throughput screening studies, where different assay systems are able to capture measurements with varying levels of precision. More specifically, $\xi_{i j k}(d, t) \sim N\left(0, \sigma_{\xi_{j}}\right)$, where $\sigma_{\xi_{j}}=.6, .8,1,1.2$ for $j=1, \ldots, 4$ respectively.

The model described in the paper was fit to the simulated surfaces. We placed relatively diffuse priors on all parameters. More precisely, we placed Gamma $(.01, .01)$ priors on the $1 / \sigma_{\epsilon_{j}}$ parameters, $\operatorname{Gamma}(1, .1)$ priors on all remaining precision parameters, and a discrete 
uniform prior on $1,2,4,8,16$, and 32 , for the degrees of freedom parameter $\nu$. The population level parameters $\alpha_{o_{i}}$ are modeled as $N(0,10)$ and the $\boldsymbol{\beta}_{\boldsymbol{o}_{\boldsymbol{i}}}$ and $\boldsymbol{\gamma}_{\boldsymbol{o}_{\boldsymbol{i}}}$ parameters as truncated $N_{4}(\mathbf{0}, 10 \boldsymbol{I})$. We fixed $\beta_{i j 2}=0, \gamma_{i j 2}=0$, and $\delta_{i j 2}=0$, assuming no effect before the first change-point. Note that this constraint can be changed to $\beta_{i j 2} \leq 0, \gamma_{i j 2} \leq 0$ and $\delta_{i j 2} \leq 0$, when assuming a tonic effect before the first change-point. We also placed a diffuse $N_{4}(\mathbf{0}, 100 \boldsymbol{I})$ prior on our dose-time interaction amplitude parameters $\boldsymbol{\delta}_{i j}$. Finally, in order to assess the sensitivity of the model results to our choice of prior parameters, we specified increasingly informative priors on our population level change-point parameters $\boldsymbol{\lambda}_{\phi_{i}}$ and $\boldsymbol{\lambda}_{\phi_{i}}$, and on our dose-time interaction change-point parameters $\chi_{i j}$. Our inferences are based on 20,000 MCMC samples, after discarding a conservative 60,000 iterations for burn-in.

Figure 1 gives examples of increasingly informative prior distributions on our dose-response change-point parameters $\phi_{i j 1}$ and $\phi_{i j 2}$. We impose a right-skewed prior distribution on $\phi_{i j 1}$, which favors (a-priori) conservative values for the location of the first change-point parameter, and allow a relatively diffuse prior distribution for our second change-point parameter $\phi_{i j 2}$. Similar examples can be constructed for our duration-response change-points $\psi_{i j 1}$ and $\psi_{i j 2}$, and our dose-time interaction change-points $\chi_{i j 1}$ and $\chi_{i j 2}$.

Figures 2 through 5 provide results from our simulation study using increasingly informative priors on our population level change-point parameters $\boldsymbol{\lambda}_{\phi_{i}}$ and $\boldsymbol{\lambda}_{\psi_{i}}$, and our dose-time interaction change-point parameters $\boldsymbol{\chi}_{i j}$. Simulated dose (column 2), duration (column 3), and dose-time interaction (column 4) response curves are superimposed with expected dose and duration-response curves, and point-wise 95\% posterior intervals estimated from our model. Prior 1 (blue) is the least informative, and corresponds to a set of relatively diffuse $\operatorname{Gamma}(2,1)$ and $\operatorname{Gamma}(3,1)$ priors on $\boldsymbol{\lambda}_{\phi_{\boldsymbol{i}}}$ and $\boldsymbol{\lambda}_{\phi_{\boldsymbol{i}}}$, and a diffuse $B_{2}(2,3,1,1, D T)$ prior on $\boldsymbol{\chi}_{i j}$. Prior 2 is a moderately informative $\operatorname{Gamma}(2.5,1)$ and $\operatorname{Gamma}(7,1.5)$ prior on $\boldsymbol{\lambda}_{\phi_{i}}$ and $\boldsymbol{\lambda}_{\phi_{i}}$, and a $B_{2}(2.5,4.7,1,1, D T)$ prior on $\boldsymbol{\chi}_{i j}$. Finally prior 3 is the most informa- 
tive, and corresponds to a $\operatorname{Gamma}(2,1)$ and $\operatorname{Gamma}(7,1.5)$ prior on $\boldsymbol{\lambda}_{\phi_{i}}$ and $\boldsymbol{\lambda}_{\phi_{i}}$, and a $B_{2}(2,4.7,1,1, D T)$ prior on $\boldsymbol{\chi}_{i j}$. The left panel shows fitted dose-response-surfaces, spanning dose and time, for our least informative prior. Table 1 also provides posterior mean estimates of $\rho_{i j}$, which are the expected inclusion probabilities of the dose-time interaction function, after adjusting for multiplicity, and can be used to test for a dose-time interaction.

The simulation results show that the posterior mean response trajectories are able to adequately estimate the true dose and duration-response curves, while capturing the most important features. In particular, our model is able to appropriately model both canonical and non-canonical dose-response trajectories, while providing interpretable risk assessment parameters. It is also evident that our model is relatively insensitive to the choice of prior parameters for our change-points. Priors 1-3 are, in most cases, indistinguishable, especially in those cases where the data sufficiently provide information on the location of the changepoints. Finally, although we do not include a formal simulation study to determine the false positive and negative rate for correctly identifying an interaction function using our model, we do notice that in all the simulated cases the model is able to correctly distinguish between data simulated with and without a dose*time interaction function. 

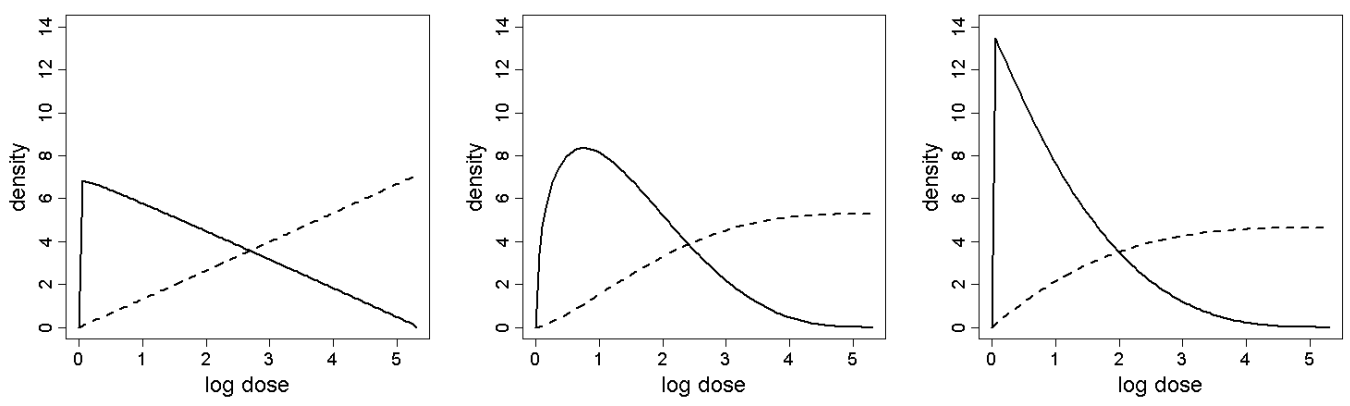

Figure 1: Marginal prior distributions on the change-point parameters. Examples of increasingly informative prior distributions on the change-point parameters $\phi_{i j 1}, \phi_{i j 2}$. We favor (a-priori) the choice of conservative values for the location of the first change-point (solid line) and a relatively diffuse prior for our second change-point (dotted line).

\begin{tabular}{cccccc}
\hline \hline Prior & ENM & Outcome 1 & Outcome 2 & Outcome 3 & Outcome 4 \\
\hline \multirow{4}{*}{ Prior1 } & 1 & 0.01 & 0.99 & 0.99 & 0.00 \\
& 2 & 0.00 & 0.00 & 0.99 & 0.99 \\
& 3 & 0.99 & 0.00 & 0.99 & 0.00 \\
& 4 & 0.99 & 0.99 & 0.01 & 0.00 \\
\hline \multirow{4}{*}{ Prior2 } & 1 & 0.00 & 0.98 & 0.99 & 0.00 \\
& 2 & 0.00 & 0.00 & 0.99 & 0.99 \\
& 3 & 0.99 & 0.00 & 0.99 & 0.00 \\
Prior3 & 4 & 0.99 & 0.99 & 0.00 & 0.00 \\
& 1 & 0.00 & 0.99 & 0.99 & 0.00 \\
& 2 & 0.00 & 0.01 & 0.99 & 0.99 \\
& 3 & 0.99 & 0.00 & 0.99 & 0.11 \\
\hline
\end{tabular}

Table 1: Expected inclusion probabilities of the dose-time interaction function using priors 1-3. Posterior mean estimates of $\rho_{i j}$ describe the expected inclusion probabilities of the dose-time interaction function, and can be used to test for an interaction. 

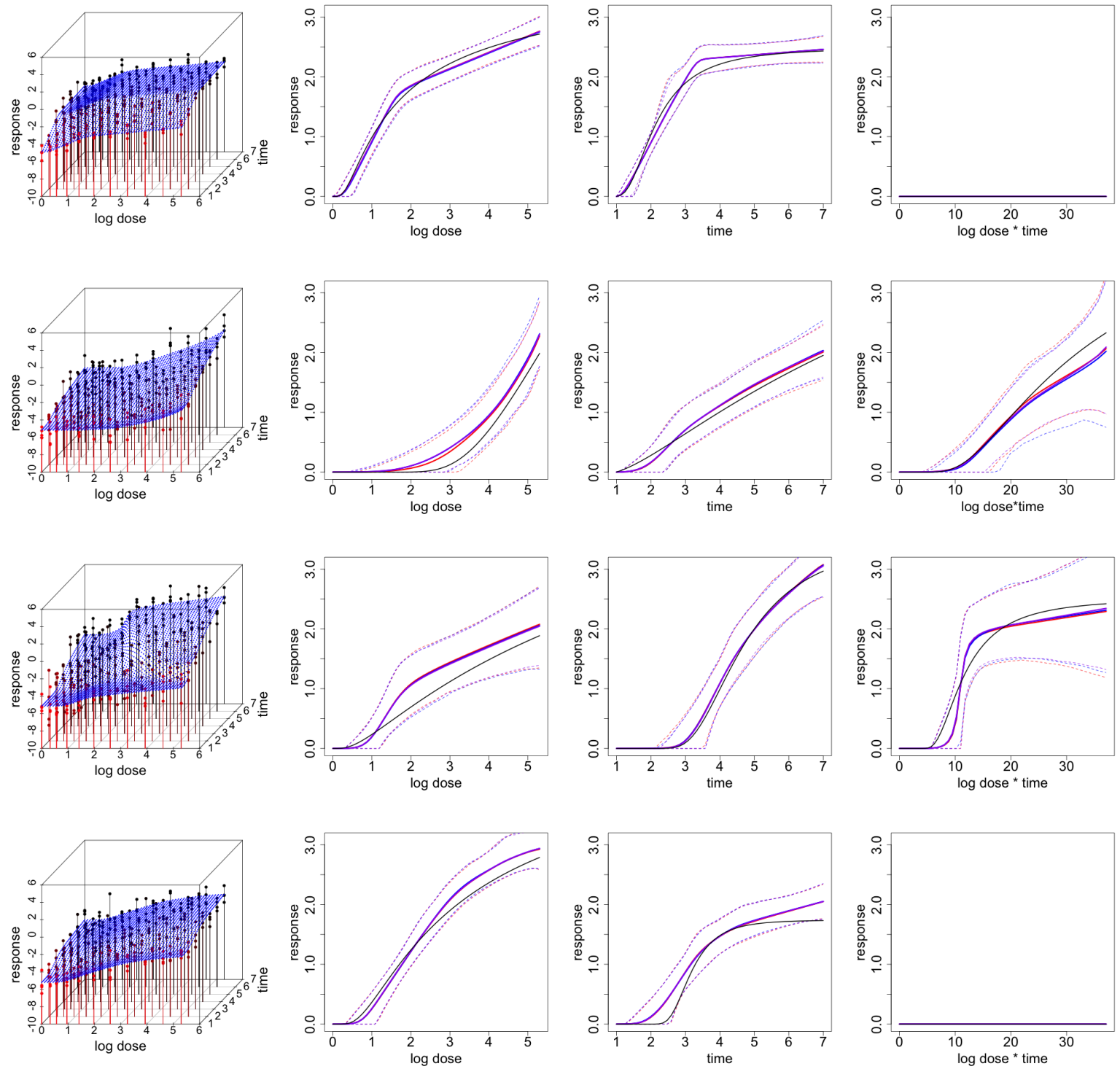

Figure 2: Simulation study to assess model fit and prior sensitivity (weibull) (column 1) Fitted response-surfaces estimated using prior 1, superimposed with simulated data. Simulated dose (column 2), duration (column 3), and dose-time interaction (column 4) response curves (black) superimposed with expected curves estimated from our model (solid colored lines) and point-wise 95\% posterior intervals (dashed lines). Prior 1 (blue) is the least informative, prior 2 (red) is moderately informative and priors 3 (purple) is the most informative. (column 1) Fitted response-surfaces estimated using prior 1, superimposed with simulated data. 

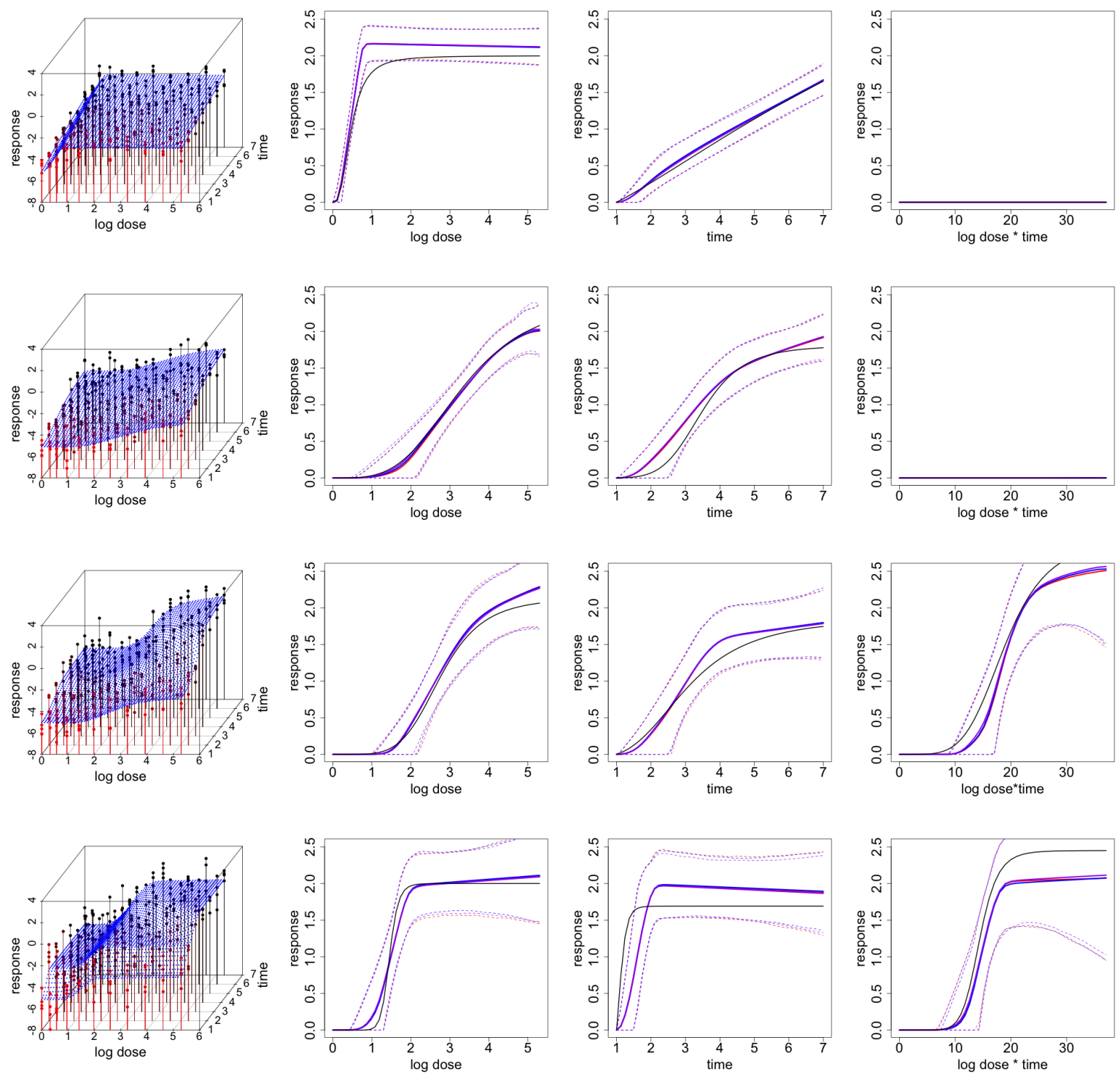

Figure 3: Simulation study to assess model fit and prior sensitivity (log-logistic) (column 1) Fitted response-surfaces estimated using prior 1, superimposed with simulated data. Simulated dose (column 2), duration (column 3), and dose-time interaction (column 4) response curves (black) superimposed with expected curves estimated from our model (solid colored lines) and point-wise 95\% posterior intervals (dashed lines). Prior 1 (blue) is the least informative, prior 2 (red) is moderately informative and priors 3 (purple) is the most informative. (column 1) Fitted response-surfaces estimated using prior 1, superimposed with simulated data. 

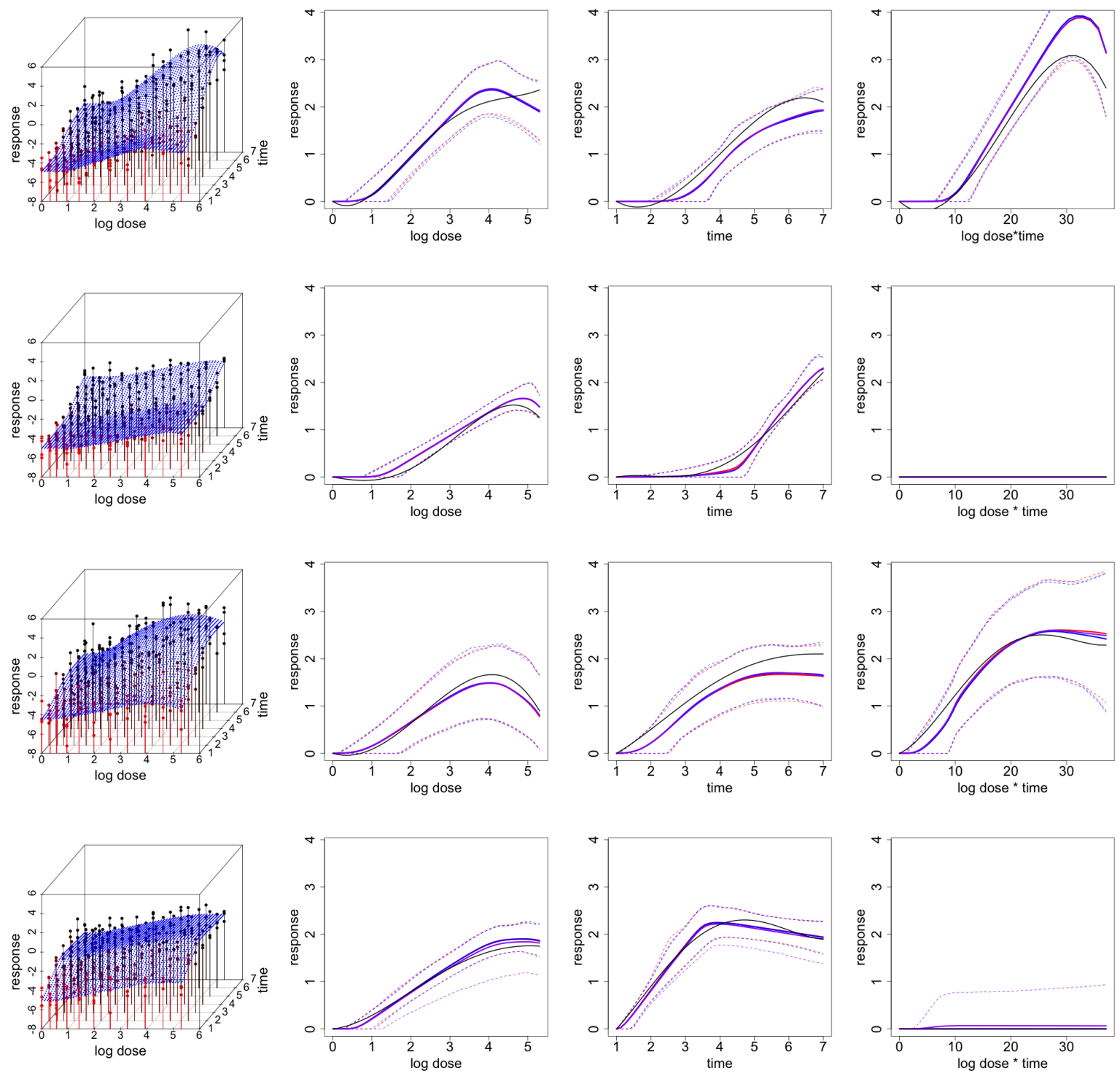

Figure 4: Simulation study to assess model fit and prior sensitivity (polynomial) (column 1) Fitted response-surfaces estimated using prior 1, superimposed with simulated data. Simulated dose (column 2), duration (column 3), and dose-time interaction (column 4) response curves (black) superimposed with expected curves estimated from our model (solid colored lines) and point-wise 95\% posterior intervals (dashed lines). Prior 1 (blue) is the least informative, prior 2 (red) is moderately informative and priors 3 (purple) is the most informative. (column 1) Fitted response-surfaces estimated using prior 1, superimposed with simulated data. 

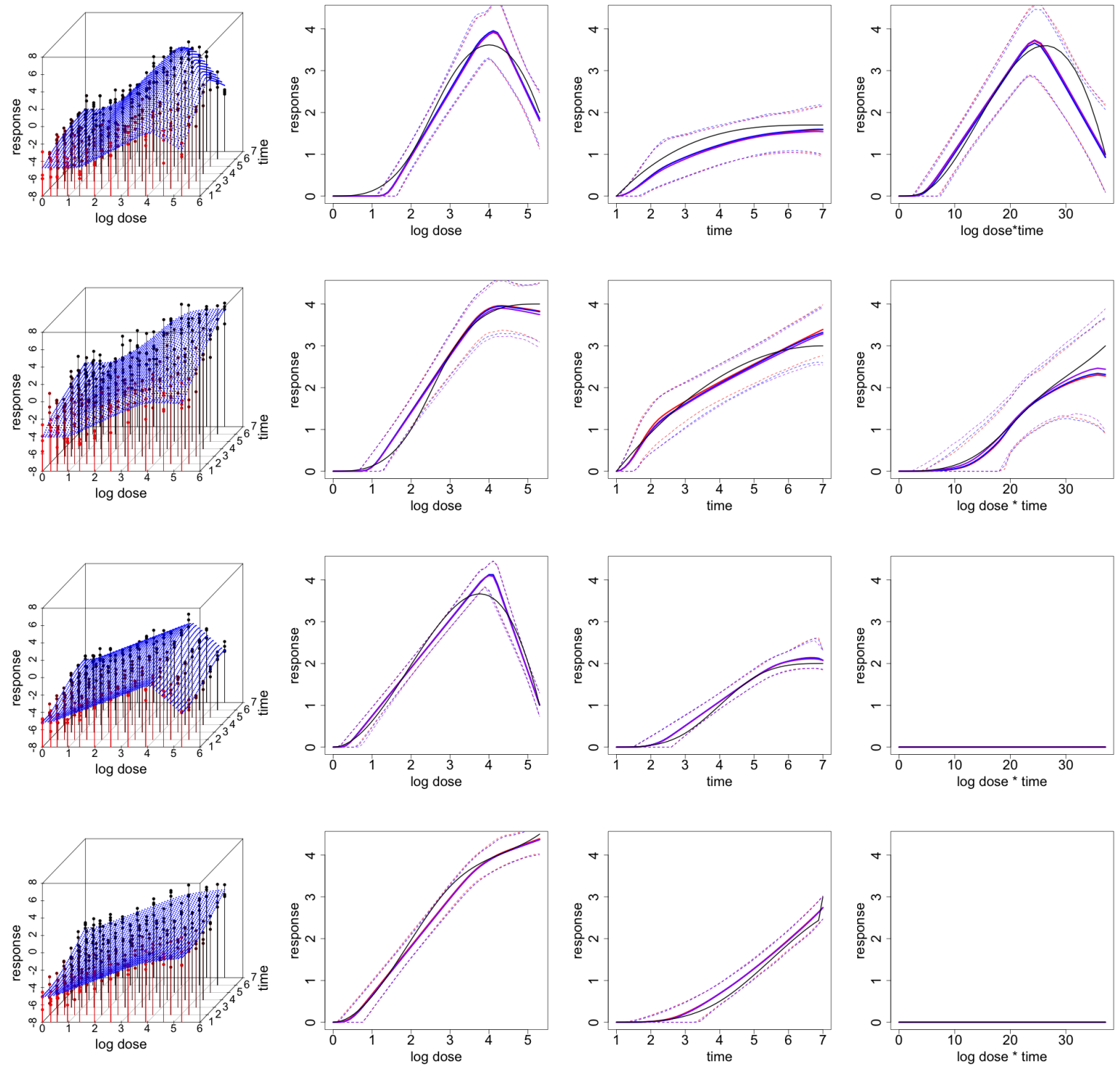

Figure 5: Simulation study to assess model fit and prior sensitivity (cubic splines) (column 1) Fitted response-surfaces estimated using prior 1, superimposed with simulated data. Simulated dose (column 2), duration (column 3), and dose-time interaction (column 4) response curves (black) superimposed with expected curves estimated from our model (solid colored lines) and point-wise 95\% posterior intervals (dashed lines). Prior 1 (blue) is the least informative, prior 2 (red) is moderately informative and priors 3 (purple) is the most informative. (column 1) Fitted response-surfaces estimated using prior 1, superimposed with simulated data. 


\section{Appendix C: Simulation Study Assessing Prior Model Sensitivity}

To assess sensitivity of the model to our choice of a prior model on the change-point parameters, we conducted a sensitivity analysis using the simulated data described above. We fit the simulated data to models with three different priors on the change-point parameters. The first prior was the bivariate beta prior parameterization described above, where we impose a right-skewed prior distribution on $\phi_{i j 1}, \psi_{i j 1}$, and $\chi_{i j 1}$, and allow a conditionally uniform prior distribution for our second change-point parameters $\phi_{i j 2}, \psi_{i j 2}$, and $\chi_{i j 2}$. Here we specified a set of relatively diffuse $\operatorname{Gamma}(2,1)$ and $\operatorname{Gamma}(3,1)$ priors for our population level change-point parameters $\boldsymbol{\lambda}_{\phi_{i}}$ and $\boldsymbol{\lambda}_{\phi_{i}}$, and a diffuse $B_{2}(2,3,1,1, D T)$ prior for our dose-time interaction change-point parameter $\boldsymbol{\chi}_{i j}$. The second prior is also a bivariate beta prior, with extremely informative, perhaps unreasonable, $\operatorname{Gamma}(60,3)$ and $\operatorname{Gamma}(1000,10)$ priors for our population level change-point parameters $\boldsymbol{\lambda}_{\phi_{i}}$ and $\boldsymbol{\lambda}_{\phi_{i}}$, and a $B_{2}(20,100,1,1, D T)$ prior for our dose-time interaction change-point parameter $\chi_{i j}$.

The third prior is another parameterization of the bivariate beta distribution which allows for a uniform prior on the simlex, and can be described as follows:

$$
\begin{aligned}
& \boldsymbol{\phi}_{i j} \sim B_{2}(1,1,1,1, D), \\
& \boldsymbol{\psi}_{i j} \sim B_{2}(1,1,1,1, T), \\
& \boldsymbol{\chi}_{i j} \sim B_{2}(1,1,1,1, T),
\end{aligned}
$$

with support $\mathcal{S}\left(\phi_{i j}\right)=\left\{\left(\phi_{i j 1}, p h i_{i j 2}\right): 0<\phi_{i j 1}<\phi_{i j 2}<D\right\}$ and $\mathcal{S}\left(\boldsymbol{\psi}_{i j}\right)=\left\{\left(\psi_{i j 1}, p s i_{i j 2}\right)\right.$ : $\left.0<\psi_{i j 1}<\psi_{i j 2}<T\right\}$. The marginal prior distribution of $\phi_{i j 1}$ is uniform and the conditional prior distribution of $\phi_{i j 2}$ given $\phi_{i j 2}$ is also uniform. Similarly for $\boldsymbol{\psi}_{\boldsymbol{i j}}$ and $\boldsymbol{\chi}_{\boldsymbol{i j}}$.

Finally, the fourth prior consists of two truncated normal priors, where for each ENM $i$ 
and response $j$, we define the following prior distributions for $\boldsymbol{\phi}_{i j}, \boldsymbol{\psi}_{i j}$, and $\boldsymbol{\chi}_{i j}$.

$$
\begin{array}{ll}
\phi_{i j 1} \sim N\left(\phi_{o_{i 1}}, \sigma_{\phi_{i 1}}^{2}\right) I\left(0<\phi_{i j 1}<D\right), & \phi_{i j 2} \sim N\left(\phi_{o_{i 2}}, \sigma_{\phi_{i 2}}^{2}\right) I\left(\phi_{i j 2}<\phi_{i j 2}<D\right), \\
\psi_{i j 1} \sim N\left(\psi_{o_{i 1}}, \sigma_{\psi_{i 1}}^{2}\right) I\left(0<\psi_{i j 1}<T\right), & \psi_{i j 2} \sim N\left(\psi_{o_{i 2}}, \sigma_{\psi_{i 2}}^{2}\right) I\left(\psi_{i j 2}<\psi_{i j 2}<T\right), \\
\chi_{i j 1} \sim N\left(\frac{1}{3} D T, 100\right) I\left(0<\chi_{i j 1}<D T\right), & \chi_{i j 2} \sim N\left(\frac{2}{3} D T, 100\right) I\left(\chi_{i j 2}<\chi_{i j 2}<D T\right) .
\end{array}
$$

For each ENM $i$, we also define the following prior distributions for our population level parameters

$$
\begin{array}{ll}
\phi_{o_{i 1}} \sim N\left(\frac{1}{3} D, 10\right), & \phi_{o_{i 1}} \sim N\left(\frac{2}{3} D, 10\right), \\
\psi_{o_{i 1}} \sim N\left(\frac{1}{3} T, 10\right), & \psi_{o_{i 1}} \sim N\left(\frac{2}{3} T, 10\right),
\end{array}
$$

and similarly the following priors on our hyperparameters

$$
\begin{array}{ll}
1 / \sigma_{\phi_{i 1}}^{2} \sim \operatorname{Gamma}\left(a_{\phi_{i 1}}, b_{\phi_{i 1}}\right), & 1 / \sigma_{\phi_{i 2}}^{2} \sim \operatorname{Gamma}\left(a_{\phi_{i 2}}, b_{\phi_{i 2}}\right), \\
1 / \sigma_{\psi_{i 1}}^{2} \sim \operatorname{Gamma}\left(a_{\psi_{i 1}}, b_{\psi_{i 1}}\right), & 1 / \sigma_{\psi_{i 2}}^{2} \sim \operatorname{Gamma}\left(a_{\psi_{i 2}}, b_{\psi_{i 2}}\right) .
\end{array}
$$

For all other parameters we used relatively diffuse priors, as described in Appendix A.2. Our inferences are based on 20,000 MCMC samples, after discarding a conservative 60,000 iterations for burn-in.

Figures 6 through 9 provide results from our simulation study, using the four different prior models for our change-point parameters described above. Simulated dose (column 2), duration (column 3), and dose-time interaction (column 4) response curves are superimposed with expected dose and duration-response curves, and point-wise $95 \%$ posterior intervals estimated from our model. Finally, column 1 shows fitted dose-response-surfaces, spanning dose and time, for prior 2 .

In summary, our analysis shows that inferential results tend to be robust to the choice of prior model. Priors 1, 3, and 4 are, in most cases, indistinguishable. Prior 2, is an extremely informative prior, and therefore results do somewhat differ from the other 3 priors, although not unreasonably. The benefit of using the bivariate beta prior described in the main paper is that it reflects the biological mechanism of toxicity. More specifically, it assigns zero 
probability to zero dose and time where toxicity is not expected to occur. The bivariate beta prior also takes a conservative standpoint by assigning high probability to low doses and times of exposure, above a certain threshold. 

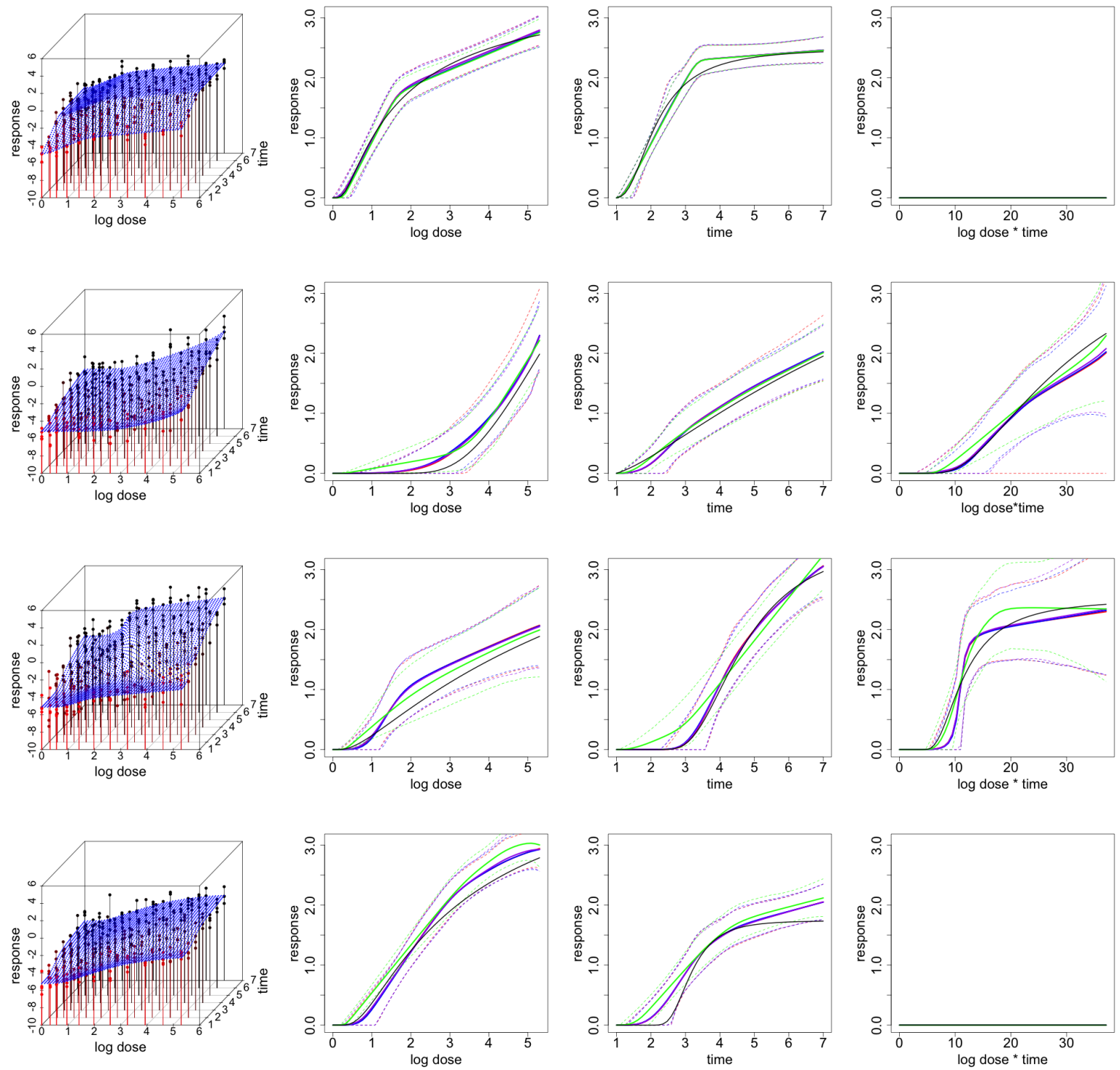

Figure 6: Simulation study to assess prior sensitivity to change-point model (weibull). (left) Fitted response-surfaces estimated using prior 2, superimposed with simulated data. Simulated dose (column 2), duration (column 3), and dose-time interaction (column 4) response curves (black) superimposed with expected curves estimated from our model (solid colored lines) and point-wise 95\% posterior intervals (dashed lines). Prior 1 (blue) is the bivariate beta prior presented in the paper, prior 2 (green) is an extremely informative bivariate beta prior, prior 3 (red) is a uniform prior over the simplex and prior 4 (purple) is the truncated normal prior described above. 

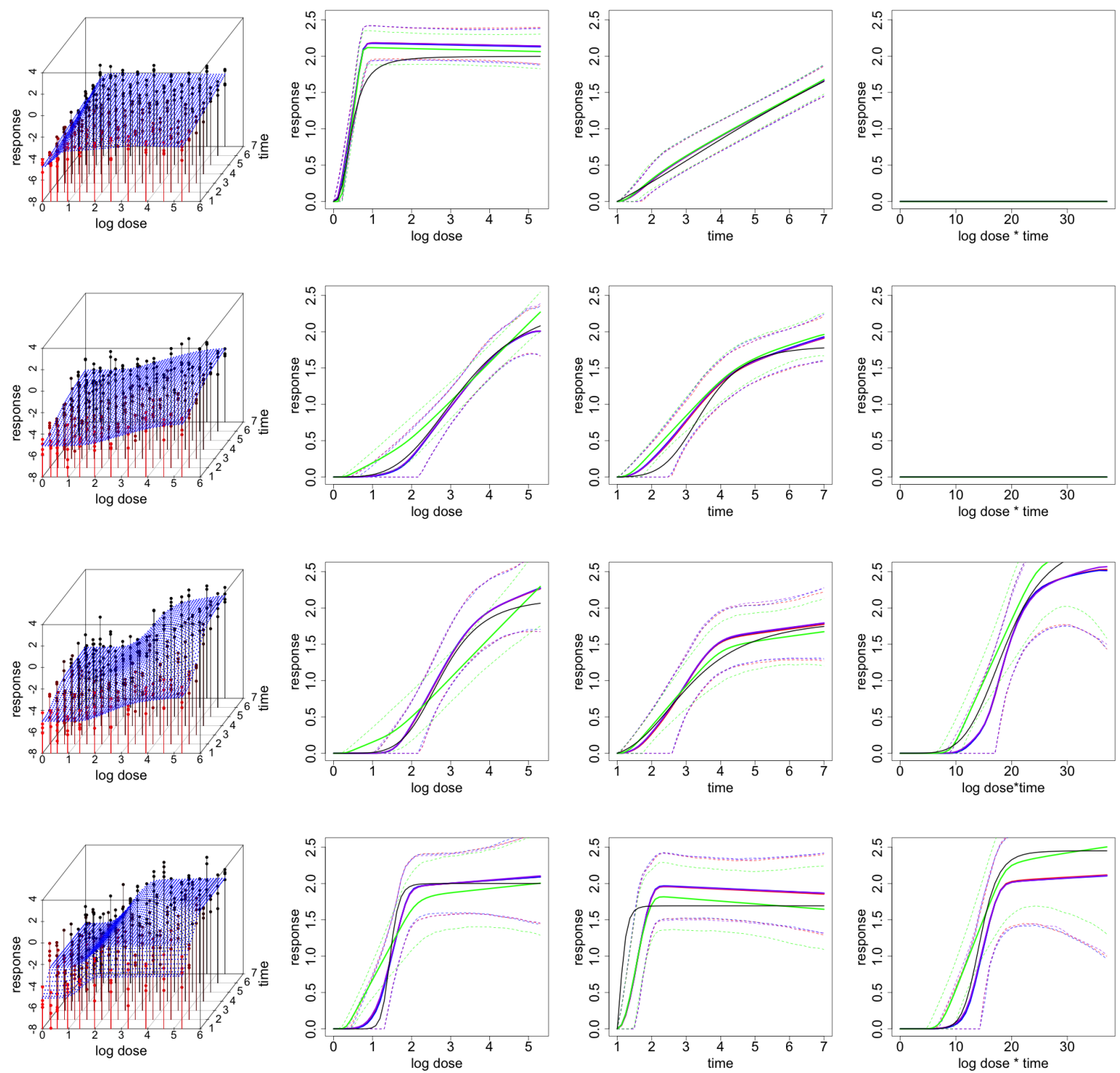

Figure 7: Simulation study to assess prior sensitivity to change-point model (loglogistic). (left) Fitted response-surfaces estimated using prior 2, superimposed with simulated data. Simulated dose (column 2), duration (column 3), and dose-time interaction (column 4) response curves (black) superimposed with expected curves estimated from our model (solid colored lines) and point-wise 95\% posterior intervals (dashed lines). Prior 1 (blue) is the bivariate beta prior presented in the paper, prior 2 (green) is an extremely informative bivariate beta prior, prior 3 (red) is a uniform prior over the simplex and prior 4 (purple) is the truncated normal prior described above. 

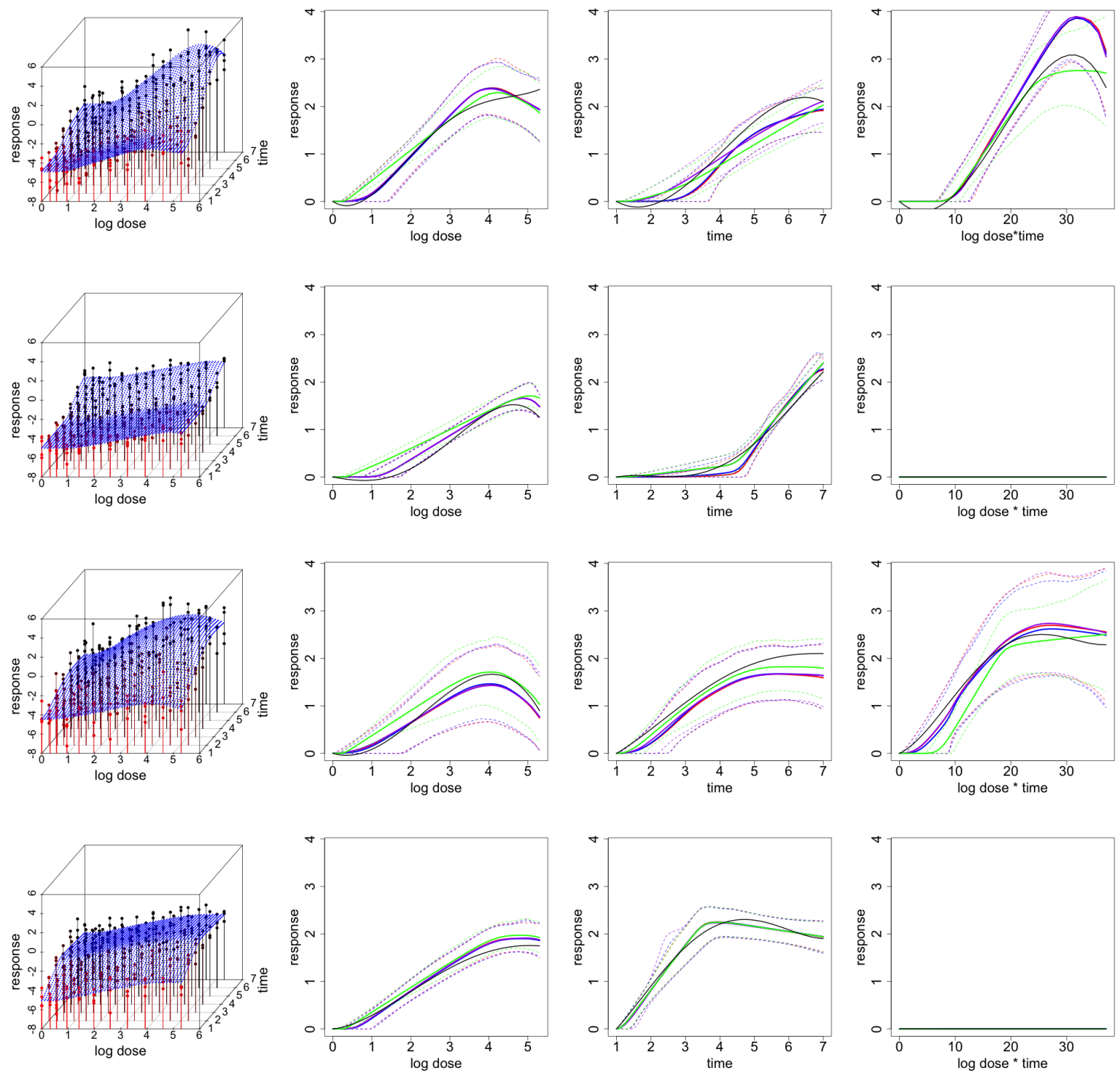

Figure 8: Simulation study to assess sensitivity to prior change-point model (polynomials). (left) Fitted response-surfaces estimated using prior 2, superimposed with simulated data. Simulated dose (column 2), duration (column 3), and dose-time interaction (column 4) response curves (black) superimposed with expected curves estimated from our model (solid colored lines) and point-wise 95\% posterior intervals (dashed lines). Prior 1 (blue) is the bivariate beta prior presented in the paper, prior 2 (green) is an extremely informative bivariate beta prior, prior 3 (red) is a uniform prior over the simplex and prior 4 (purple) is the truncated normal prior described above. 

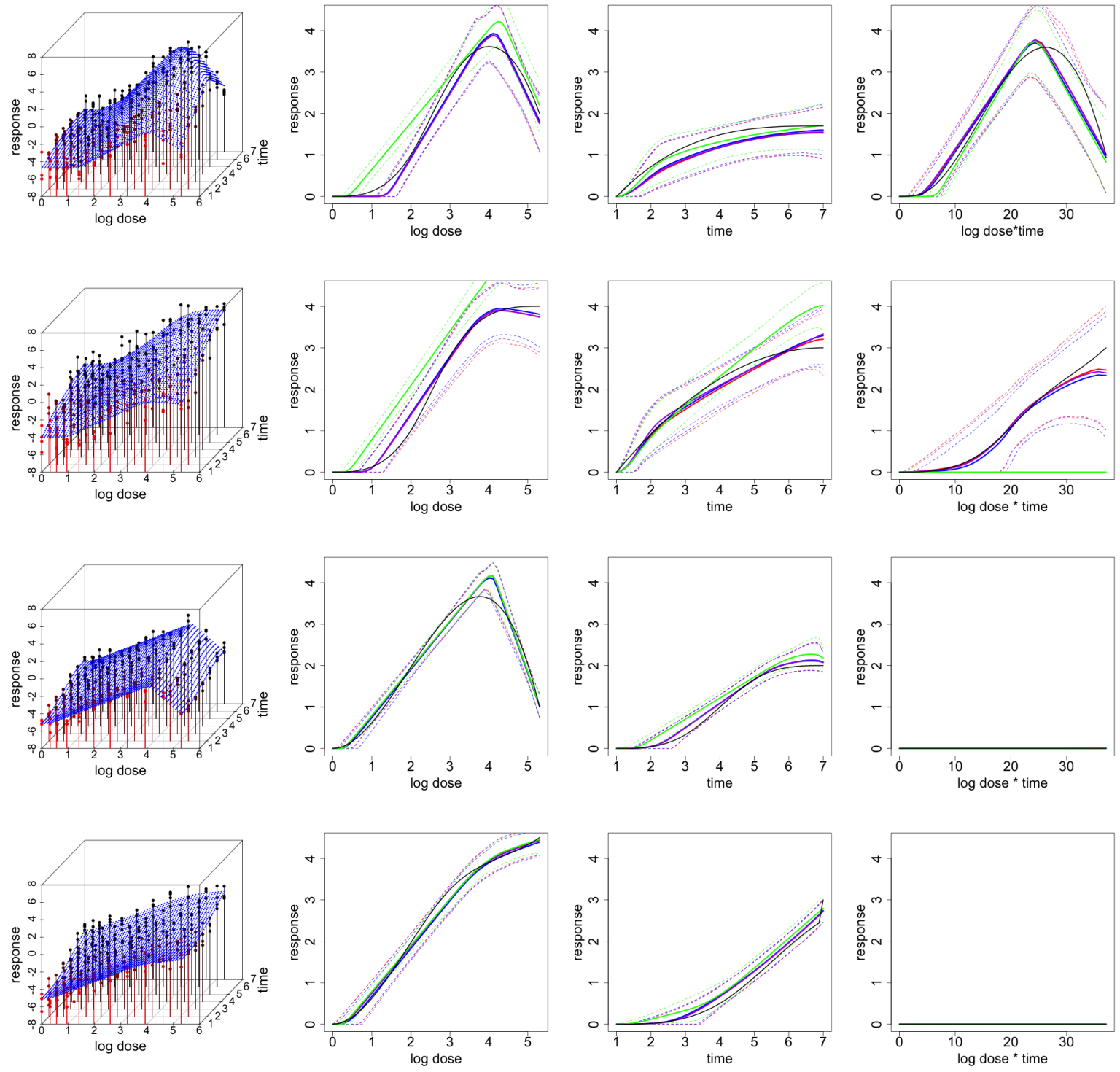

Figure 9: Simulation study to assess prior change-point model sensitivity (cubic splines. (left) Fitted response-surfaces estimated using prior 2, superimposed with simulated data. Simulated dose (column 2), duration (column 3), and dose-time interaction (column 4) response curves (black) superimposed with expected curves estimated from our model (solid colored lines) and point-wise 95\% posterior intervals (dashed lines). Prior 1 (blue) is the bivariate beta prior presented in the paper, prior 2 (green) is an extremely informative bivariate beta prior, prior 3 (red) is a uniform prior over the simplex and prior 4 (purple) is the truncated normal prior described above. 


\section{Appendix D: Figures and TABles}

The model presented in the paper was fit to data on eight metal and metal oxide nanoparticles, monitored in relation to four cytotoxicity parameters which, were measured over a grid of ten doses and seven times (hours) of exposure. The four responses include mitochondrial superoxide formation (MSF), loss of mitochondrial membrane potential (MMP), elevated intracellular calcium (EIC), and cellular membrane damage (CMD). Each outcome was measured as the percentage of cells positive for the response and was normalized using a logit transformation.

In this appendix we present figures for the remaining six particles not presented in the paper including, silver $(\mathrm{Ag})$, gold $(\mathrm{Au})$, aluminum oxide $\left(\mathrm{Al}_{2} \mathrm{O}_{3}\right)$, iron oxide $\left(\mathrm{Fe}_{3} \mathrm{O}_{4}\right)$, silicon dioxide $\left(\mathrm{SiO}_{2}\right)$, and zinc oxide ( $\left.\mathrm{ZnO}\right)$ (see Figures 10 to 15). Specifically, column 1 shows fitted dose-response surfaces spanning dose and time. Also included are fitted curves for the expected dose response function $f_{i j}(d)$ (column 2), which represent the effect due to dose, the expected duration response function $g_{i j}(t)$ (column 3), which represent the effect due to time and, the expected dose-time interaction function $h_{i j}(t)$ (column 4).

Figures 16 and 17 provides a plot of the estimated median response, relative to the background, for different doses and times of exposure. Blue colors indicate safety regions or areas of reduced risk to the cells, while red colored regions indicate increased risk of cytotoxicity. Finally, Table 2 provides posterior estimates of the expected inclusion probability of the dose-time interaction function. These probabilities can used to test for a dose-time interaction, adjusted for multiplicity. 

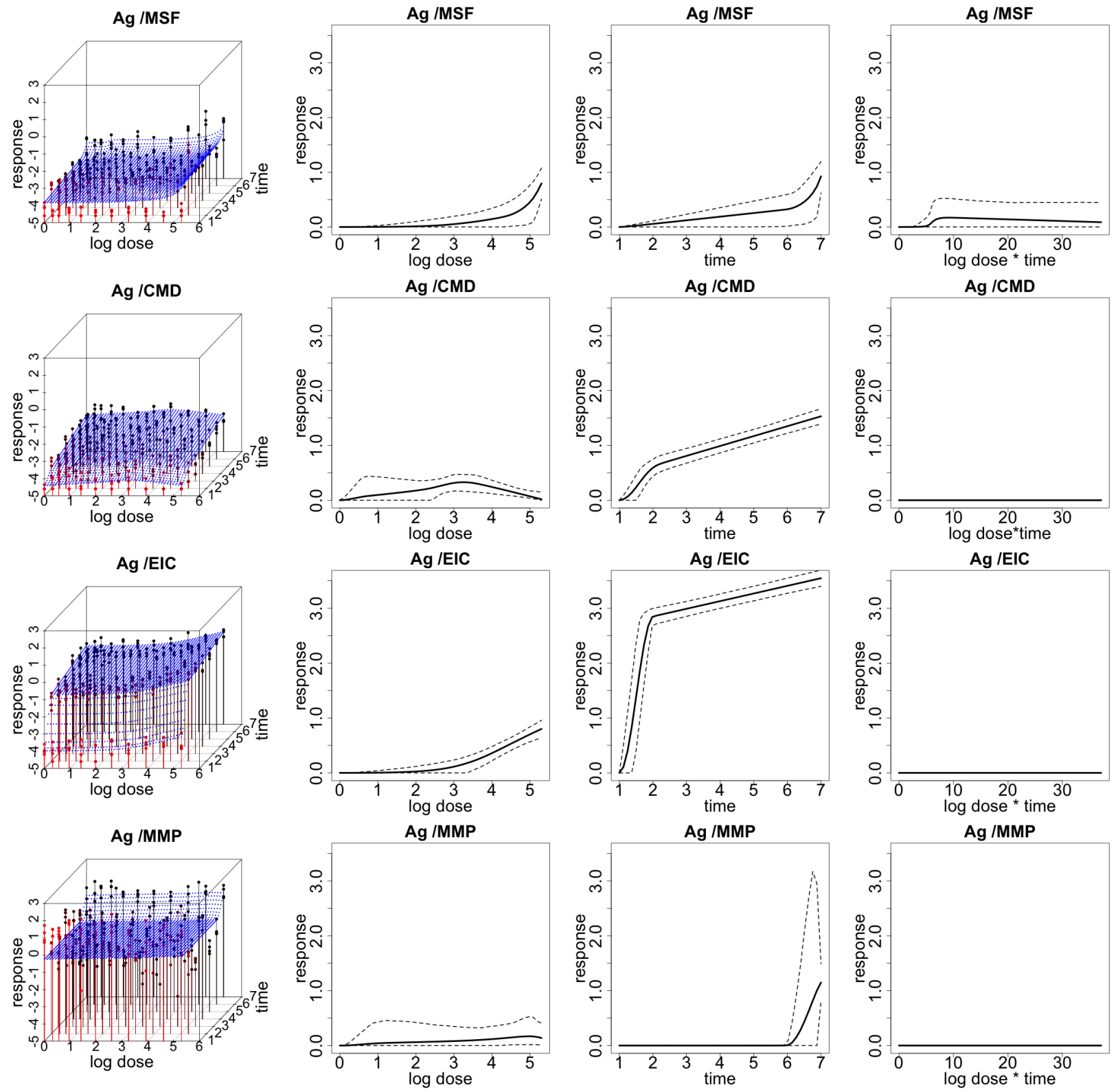

Figure 10: Fitted response curves for the silver nanomaterial (Ag). Fitted response surfaces (column 1), dose-response function, $f_{i j}(d)$ (column 2), duration-response function, $g_{i j}(t)$ (column 3), dose/duration interaction function, $h_{i j}(d t)$ (column 4) and associated $95 \%$ posterior intervals. In (column 1), the color red represents response values corresponding to lower time points and the color black represents response values corresponding to higher time points. 

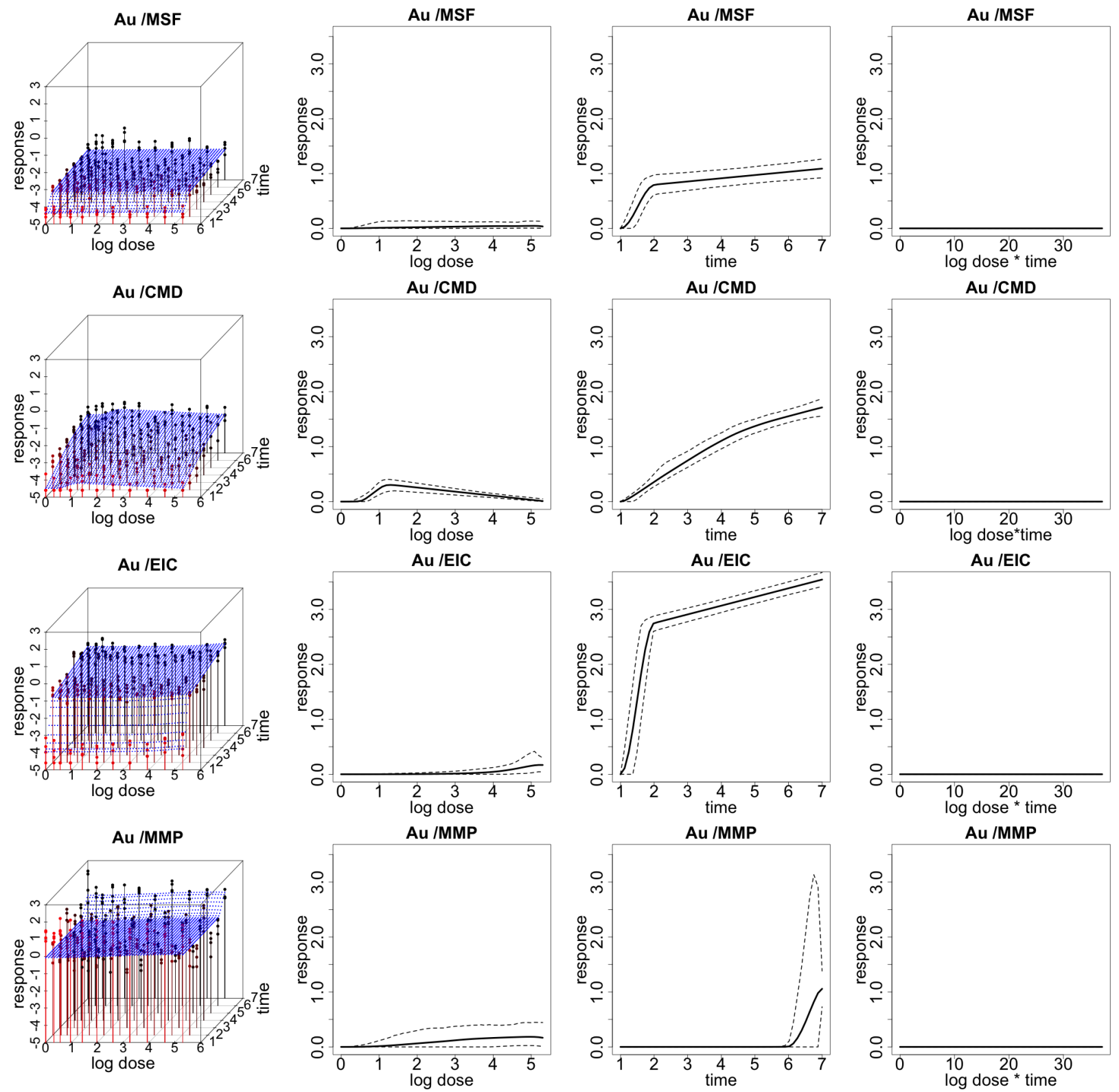

Figure 11: Fitted response curves for the gold nanomaterial $(\mathrm{Au})$. Fitted response surfaces (column 1), dose-response function, $f_{i j}(d)$ (column 2), duration-response function, $g_{i j}(t)$ (column 3), dose/duration interaction function, $h_{i j}(d t)$ (column 4) and associated $95 \%$ posterior intervals. In (column 1), the color red represents response values corresponding to lower time points and the color black represents response values corresponding to higher time points. 

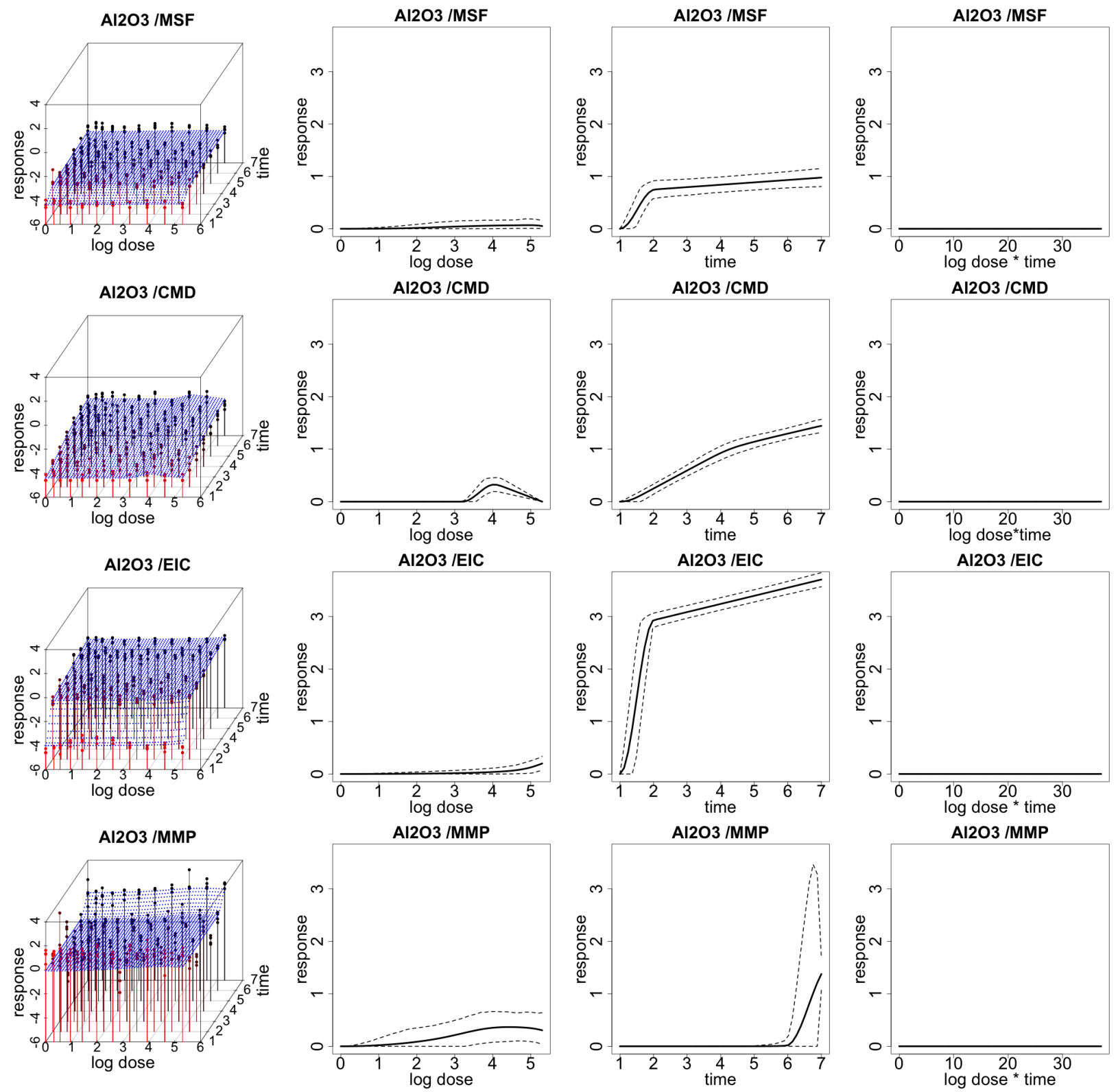

Figure 12: Fitted response curves for the aluminum oxide nanomaterial $\left(\mathrm{Al}_{2} \mathrm{O}_{3}\right)$. Fitted response surfaces (column 1), dose-response function, $f_{i j}(d)$ (column 2), durationresponse function, $g_{i j}(t)$ (column 3), dose/duration interaction function, $h_{i j}(d t)$ (column 4) and associated $95 \%$ posterior intervals. In (column 1), the color red represents response values corresponding to lower time points and the color black represents response values corresponding to higher time points. 


\begin{tabular}{lcccc}
\hline \hline ENM & MSF & EIC & CMD & MMP \\
\hline $\mathrm{Ag}$ & 0.59 & 0.00 & 0.00 & 0.99 \\
$\mathrm{Au}$ & 0.00 & 0.00 & 0.00 & 0.00 \\
$\mathrm{Pt}$ & 0.99 & 0.00 & 0.00 & 0.00 \\
$\mathrm{Al}_{2} \mathrm{O}_{3}$ & 0.00 & 0.00 & 0.00 & 0.00 \\
$\mathrm{Fe}_{3} \mathrm{O}_{4}$ & 0.99 & 0.00 & 0.00 & 0.00 \\
$\mathrm{SiO}_{2}$ & 0.99 & 0.00 & 0.00 & 0.46 \\
$\mathrm{QD}$ & 0.99 & 0.00 & 0.00 & 0.00 \\
$\mathrm{ZnO}$ & 0.99 & 0.00 & 0.99 & 0.00 \\
\hline
\end{tabular}

Table 2: Expected inclusion probability of the dose-time interaction function. Posterior mean estimates of $\rho_{i j}$ for each particle and outcome. Expected inclusion probabilities can be used to test for a dose-time interaction. 

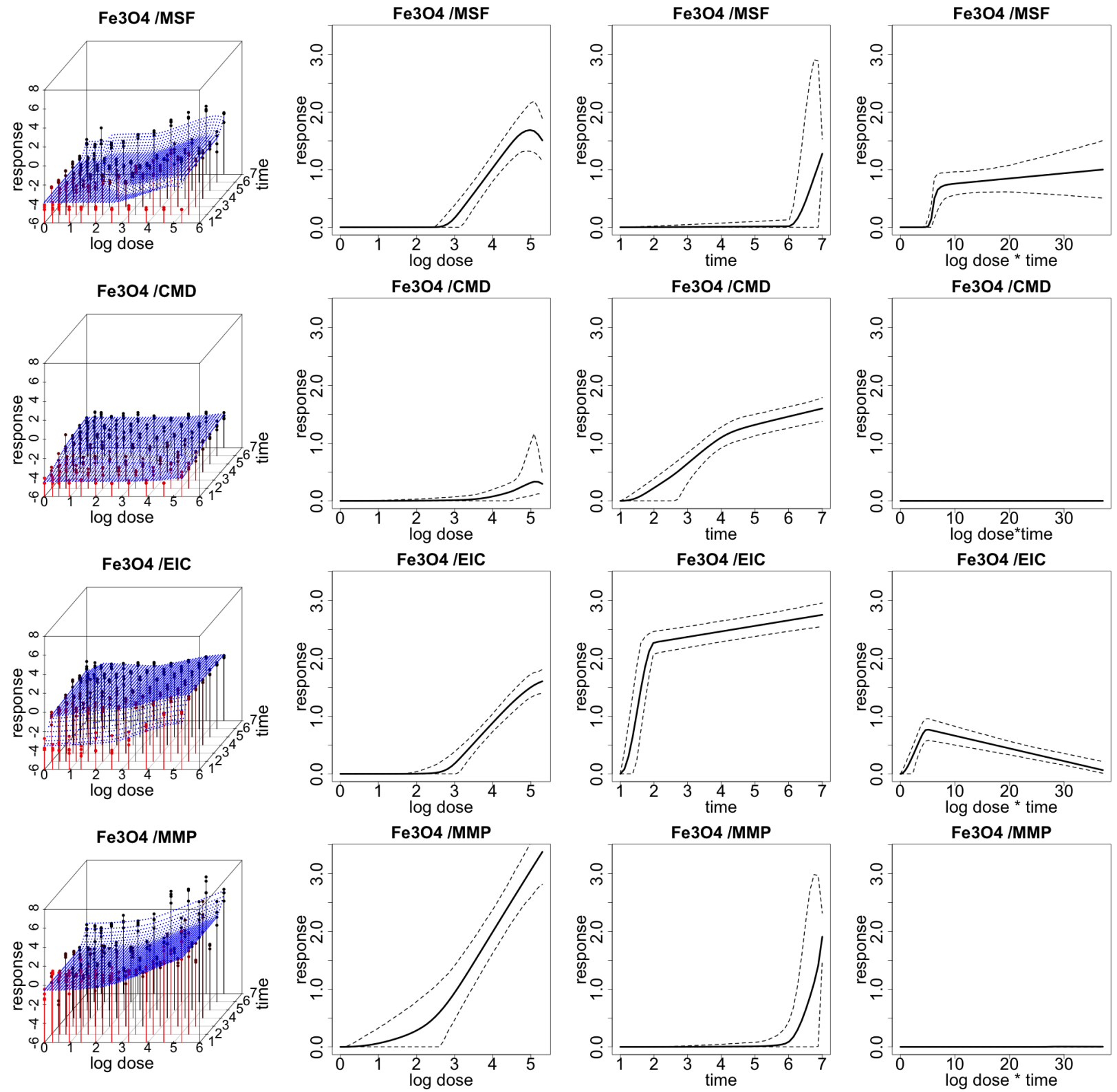

Figure 13: Fitted response curves for the iron oxide nanomaterial $\left(\mathrm{Fe}_{3} \mathrm{O}_{4}\right)$. Fitted response surfaces (column 1), dose-response function, $f_{i j}(d)$ (column 2), duration-response function, $g_{i j}(t)$ (column 3), dose/duration interaction function, $h_{i j}(d t)$ (column 4) and associated $95 \%$ posterior intervals. In (column 1), the color red represents response values corresponding to lower time points and the color black represents response values corresponding to higher time points. 

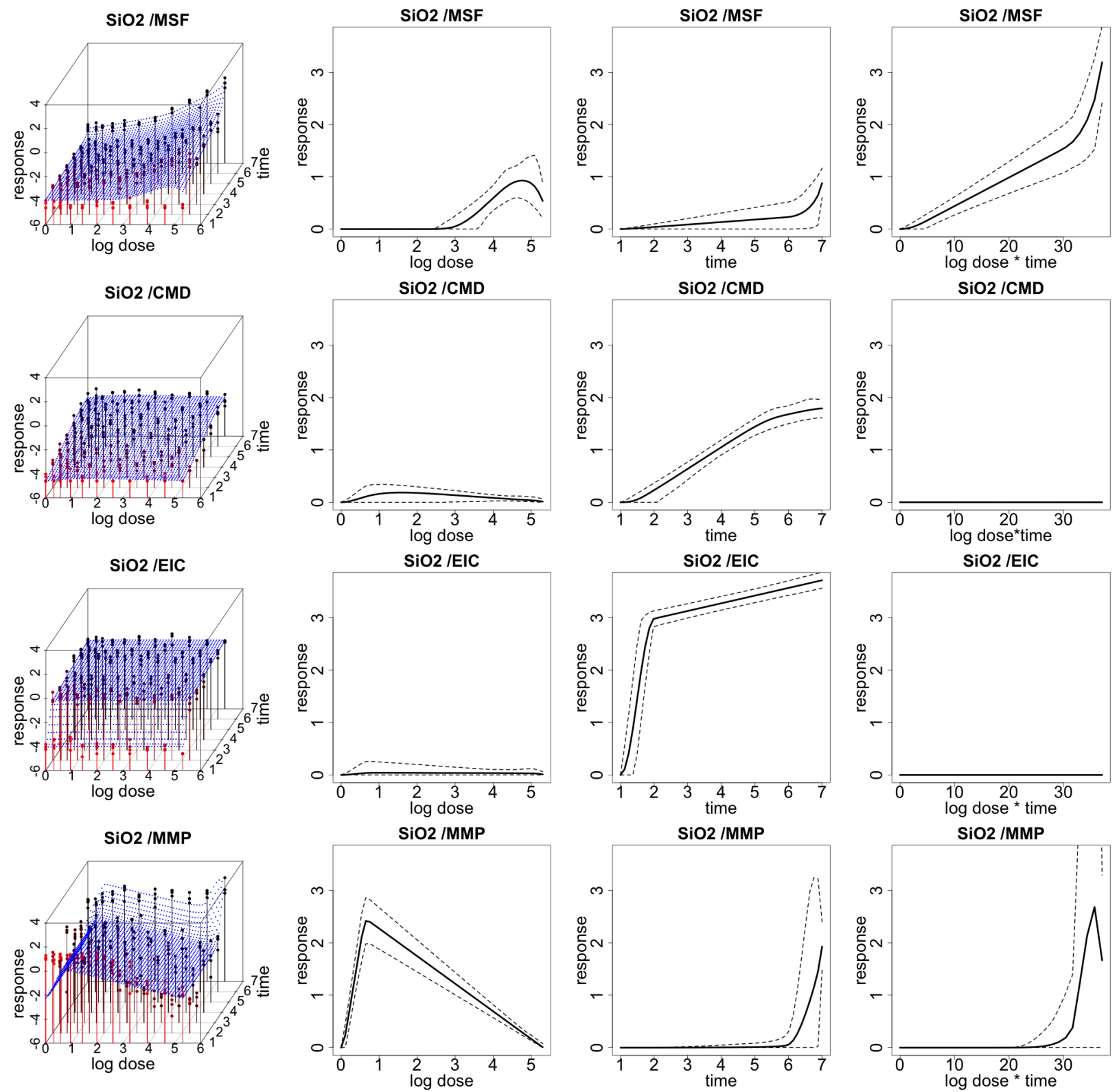

Figure 14: Fitted response curves for the silicon dioxide nanomaterial $\left(\mathrm{SiO}_{2}\right)$. Fitted response surfaces (column 1), dose-response function, $f_{i j}(d)$ (column 2), durationresponse function, $g_{i j}(t)$ (column 3), dose/duration interaction function, $h_{i j}(d t)$ (column 4) and associated $95 \%$ posterior intervals. In (column 1), the color red represents response values corresponding to lower time points and the color black represents response values corresponding to higher time points. 

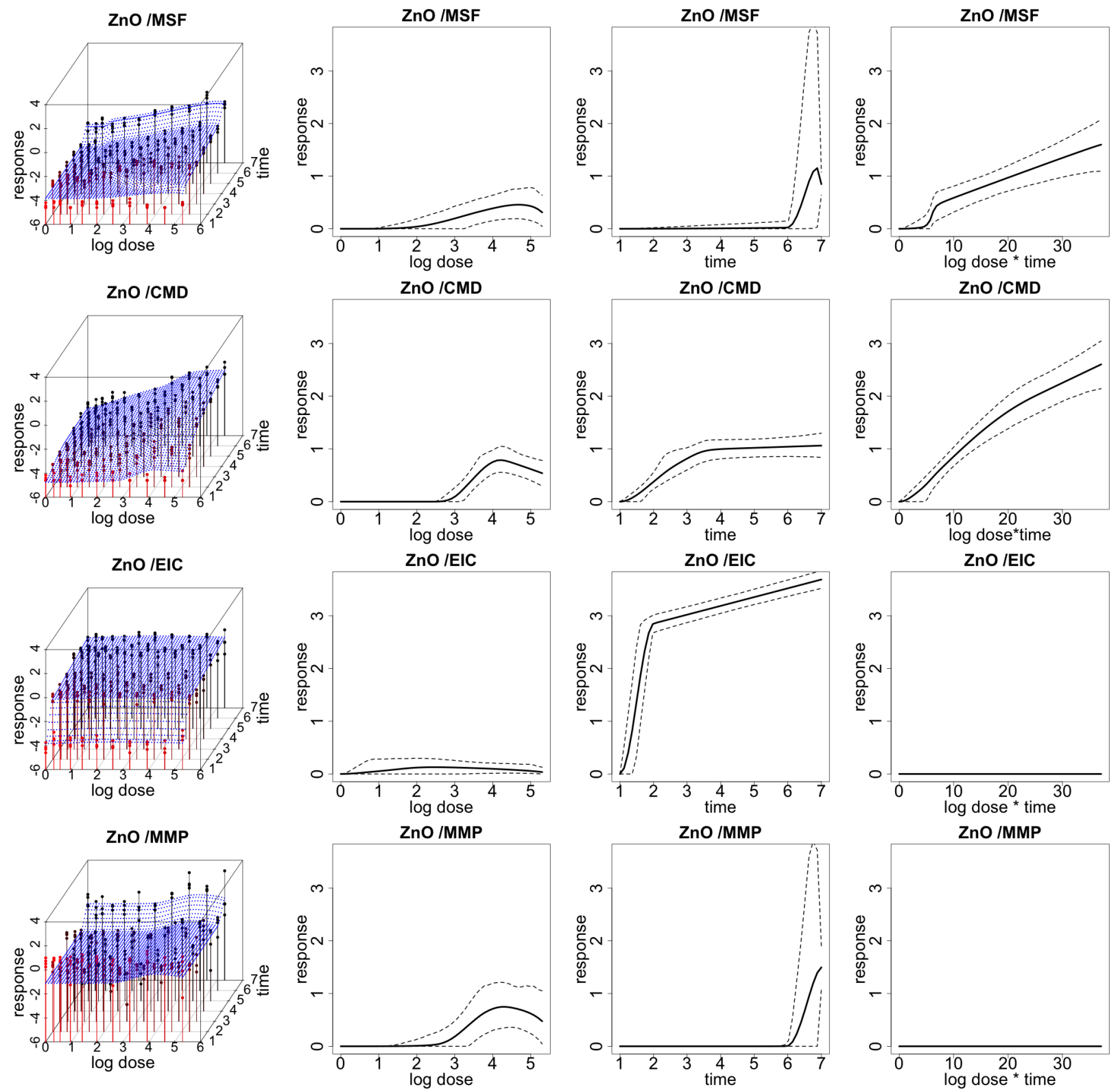

Figure 15: Fitted response curves for the quantum dot nanomaterial $(\mathrm{ZnO})$. Fitted response surfaces (column 1), dose-response function, $f_{i j}(d)$ (column 2), duration-response function, $g_{i j}(t)$ (column 3), dose/duration interaction function, $h_{i j}(d t)$ (column 4) and associated $95 \%$ posterior intervals. In (column 1), the color red represents response values corresponding to lower time points and the color black represents response values corresponding to higher time points. 

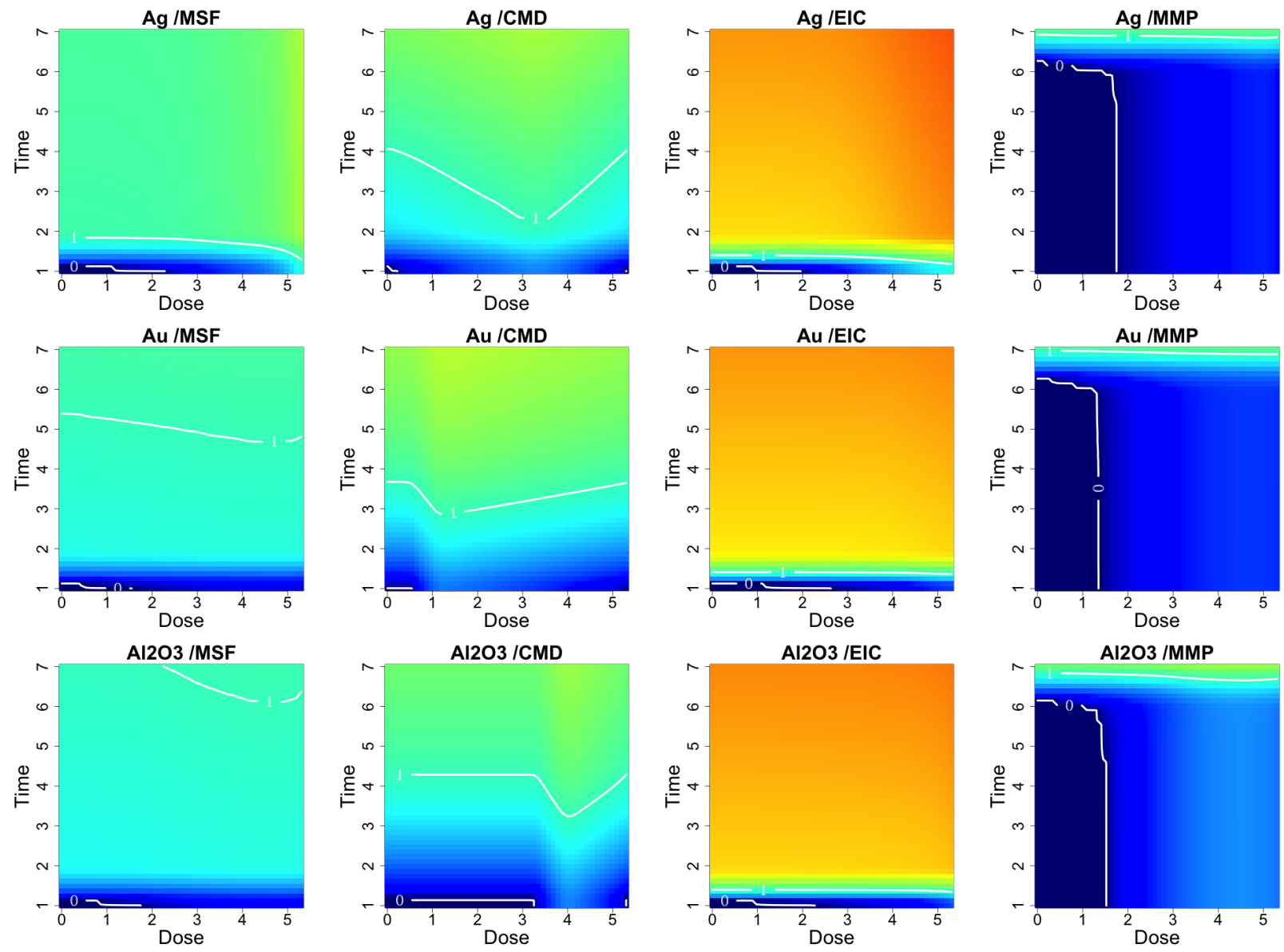

Figure 16: Safe exposure regions for $\mathbf{A g}, \mathbf{A u}$, and $\mathbf{A l}_{2} \mathbf{O}_{3}$. For each particle and outcome we can define dose and time exposure regions which do not induce cytotoxicity. Lighter regions indicate greater cytotoxicity to the cells, whereas darker regions indicate reduced risk. Contour lines quantitate the median estimated response, relative to the background, where zero response areas can be interpreted as safe exposure regions. 

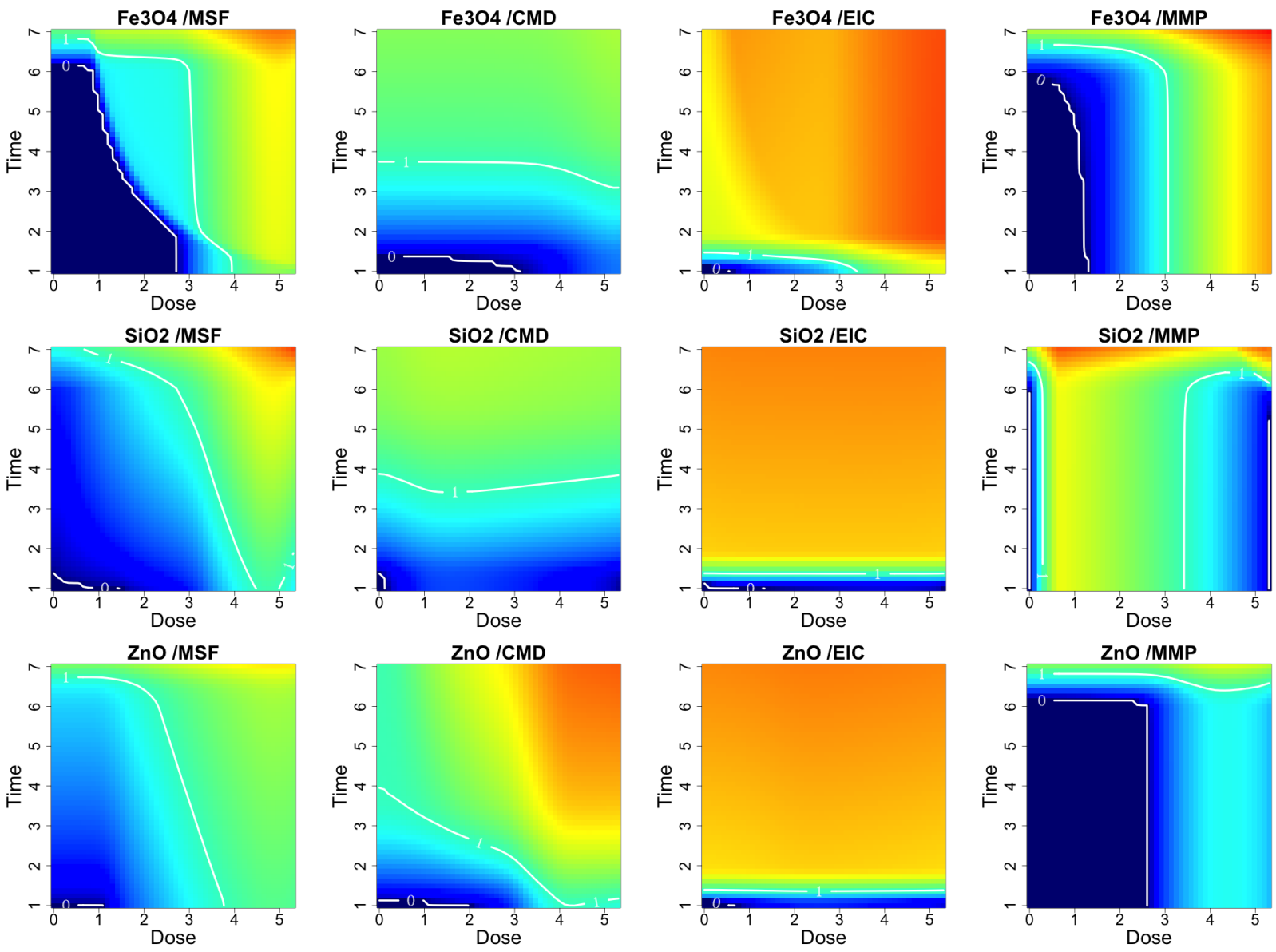

Figure 17: Safe exposure regions for $\mathrm{Fe}_{3} \mathrm{O}_{4}, \mathrm{SiO}_{2}$ and $\mathrm{ZnO}$. For each particle and outcome we can define dose and time exposure regions which do not induce cytotoxicity. Red colored regions indicate greater cytotoxicity to the cells, whereas blue colored regions indicate reduced risk. Contour lines quantitate the median estimated response, relative to the background, where zero response areas can be interpreted as safe exposure regions. 


\section{Appendix E: Model Assessment}

In this Appendix we discuss model assessment. First we include a plot of the probability integral transform (PIT) histogram as measure of predictive performance as described by Gneiting et al. (2007). Next, we check the average coverage of the posterior predictive distribution across all doses and times by plotting the distribution of the posterior predictive mean response averaged across all doses and times of exposure along with the empirical mean response across all doses and times of exposure.

Figure 19 provides a plot of the PIT histogram for the entire model, including all doses, times, outcomes, and particles. Visual assessment indicates that the plot does tend toward uniformity, indicating good overall predictive performance. Figure 19 also includes plots of the PIT histogram, separately for each ENM. The PIT histograms look approximately uniform for most ENM.

Figures 20 and 21 provide plots of the distribution of the posterior predictive mean response averaged across all doses and times of exposure (black), for each particle and outcome. The mean and associated $95 \%$ posterior intervals for this posterior predictive distribution are marked using vertical lines (black). Also included is the empirical mean response across all doses and times of exposure (red). Figure 22, summarizes these results by plotting the mean and $95 \%$ posterior intervals of the posterior predictive mean response (black), along with the the empirical mean response across all doses and times (red), for each particle and

outcome. In all cases the empirical mean response is contained within the $95 \%$ posterior intervals of the posterior predictive mean distribution, indicating relatively good posterior coverage across all doses and times of exposure. 


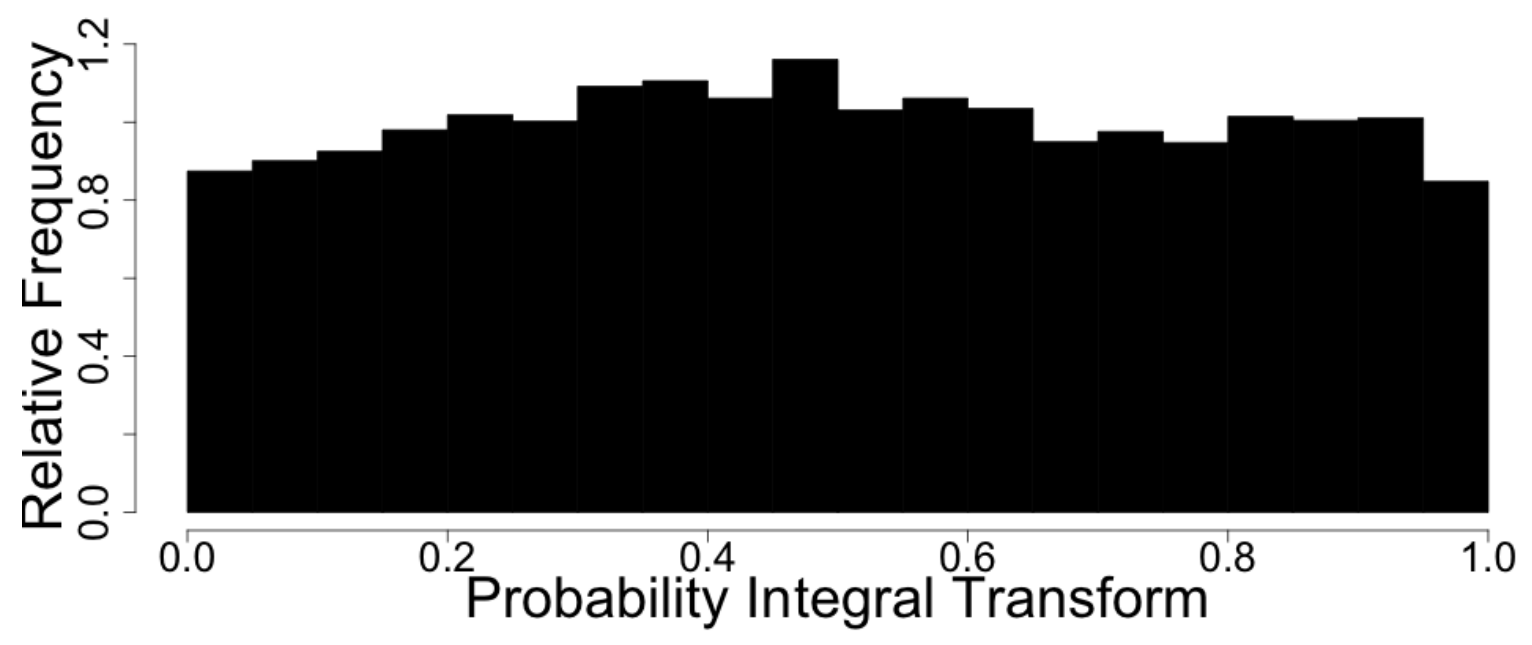

Figure 18: PIT histogram for the entire model. 

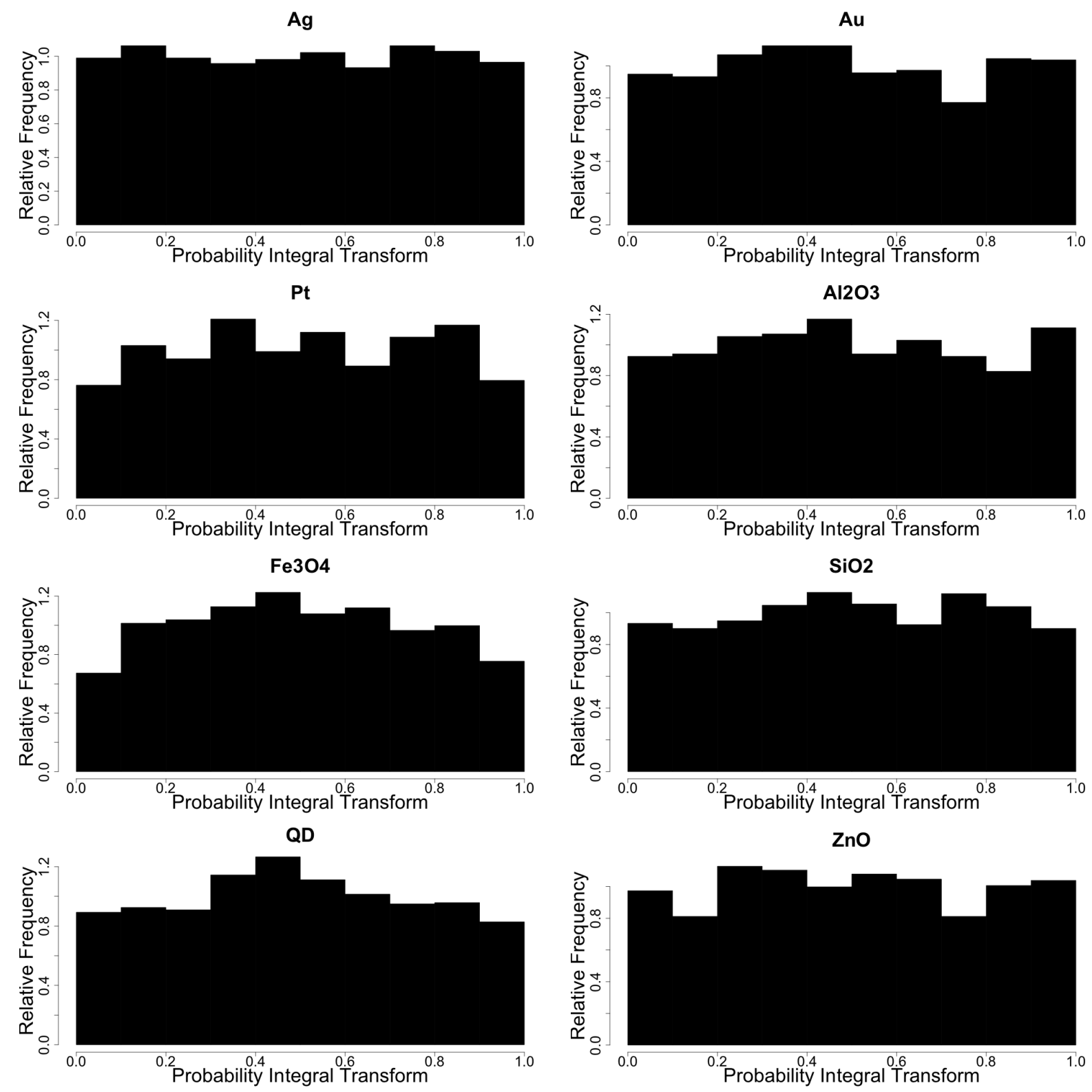

Figure 19: PIT histogram severalty for each ENM 

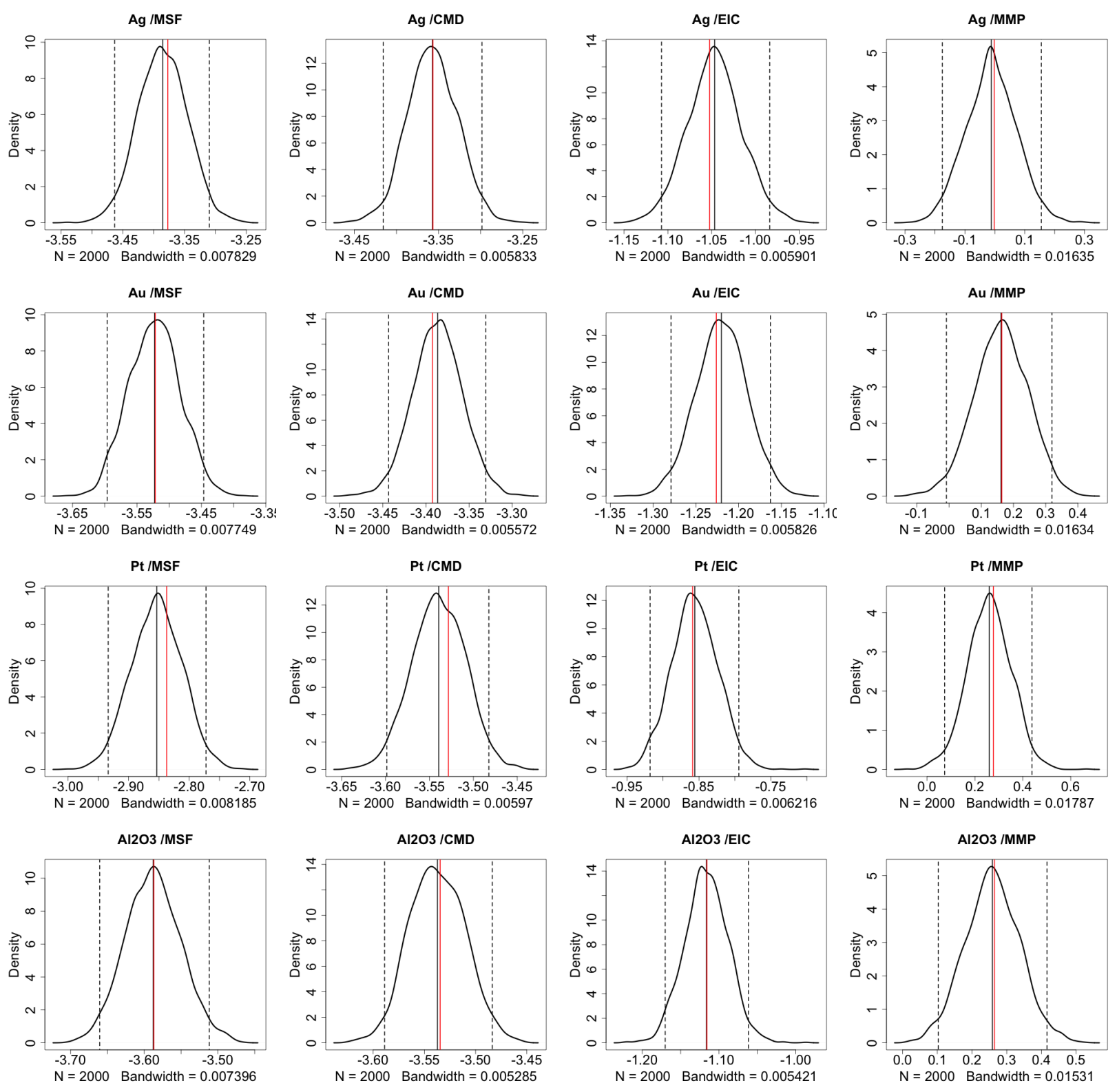

Figure 20: Posterior predictive mean distributions for $\mathrm{Ag}, \mathrm{Au}, \mathrm{Pt}$ and $\mathrm{Al}_{2} \mathrm{O}_{3}$. For each particle and outcome we plot the distribution of the posterior predictive mean response across all doses and times of exposure (black), along with the mean (solid black line) and associated 95\% posterior intervals (dotted black lines) for this distribution. Also included is the empirical mean response across all doses and times of exposure (red). 

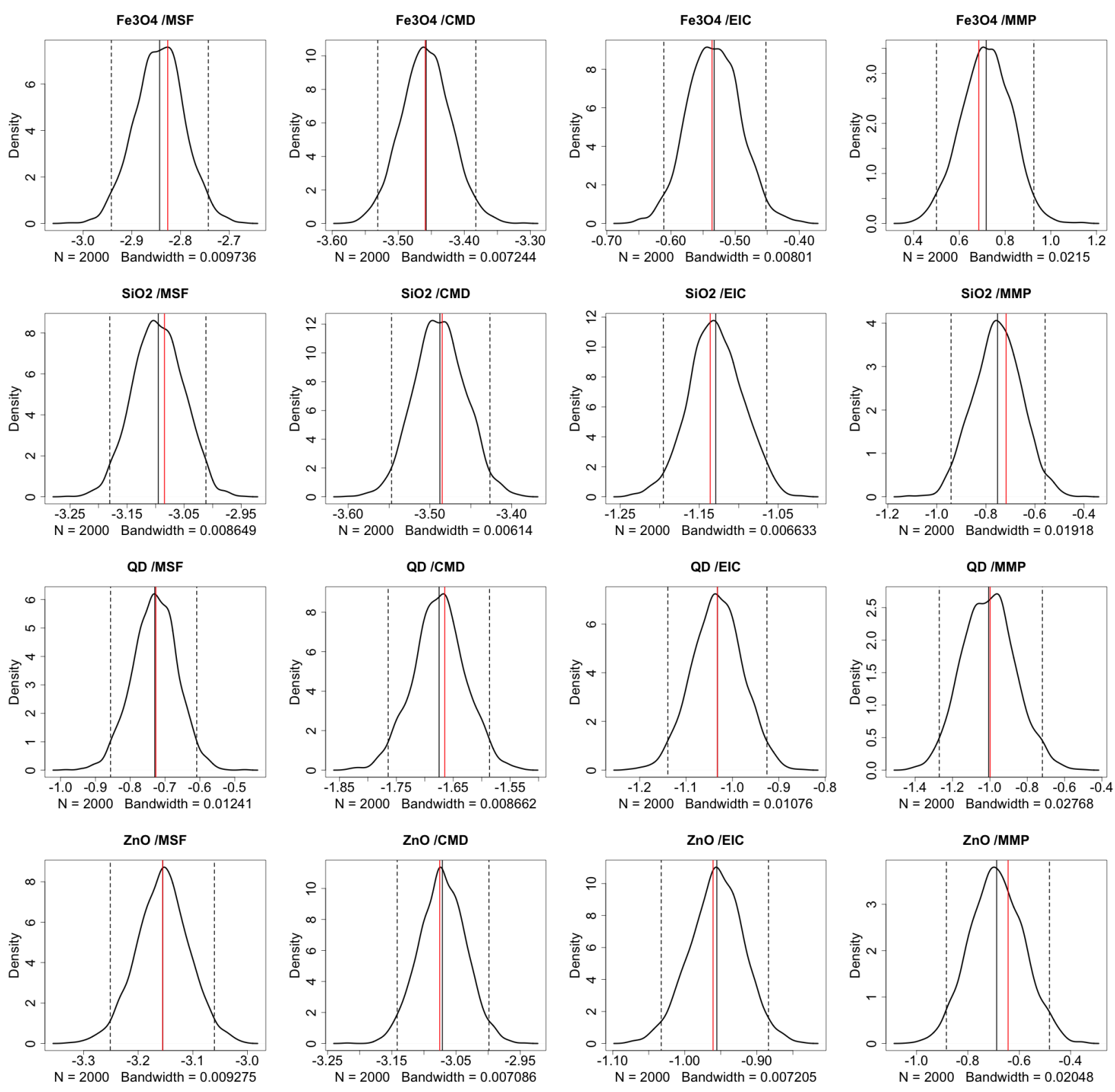

Figure 21: Posterior predictive mean distributions for $\mathrm{Fe}_{3} \mathrm{O}_{4}, \mathrm{Si} O_{2}, \mathrm{QD}$ and $\mathrm{ZnO}$. For each particle and outcome we plot the distribution of the posterior predictive mean response across all doses and times of exposure (black), along with the mean (solid black line) and associated $95 \%$ posterior intervals (dotted black lines) for this distribution. Also included is the empirical mean response across all doses and times of exposure (red). 


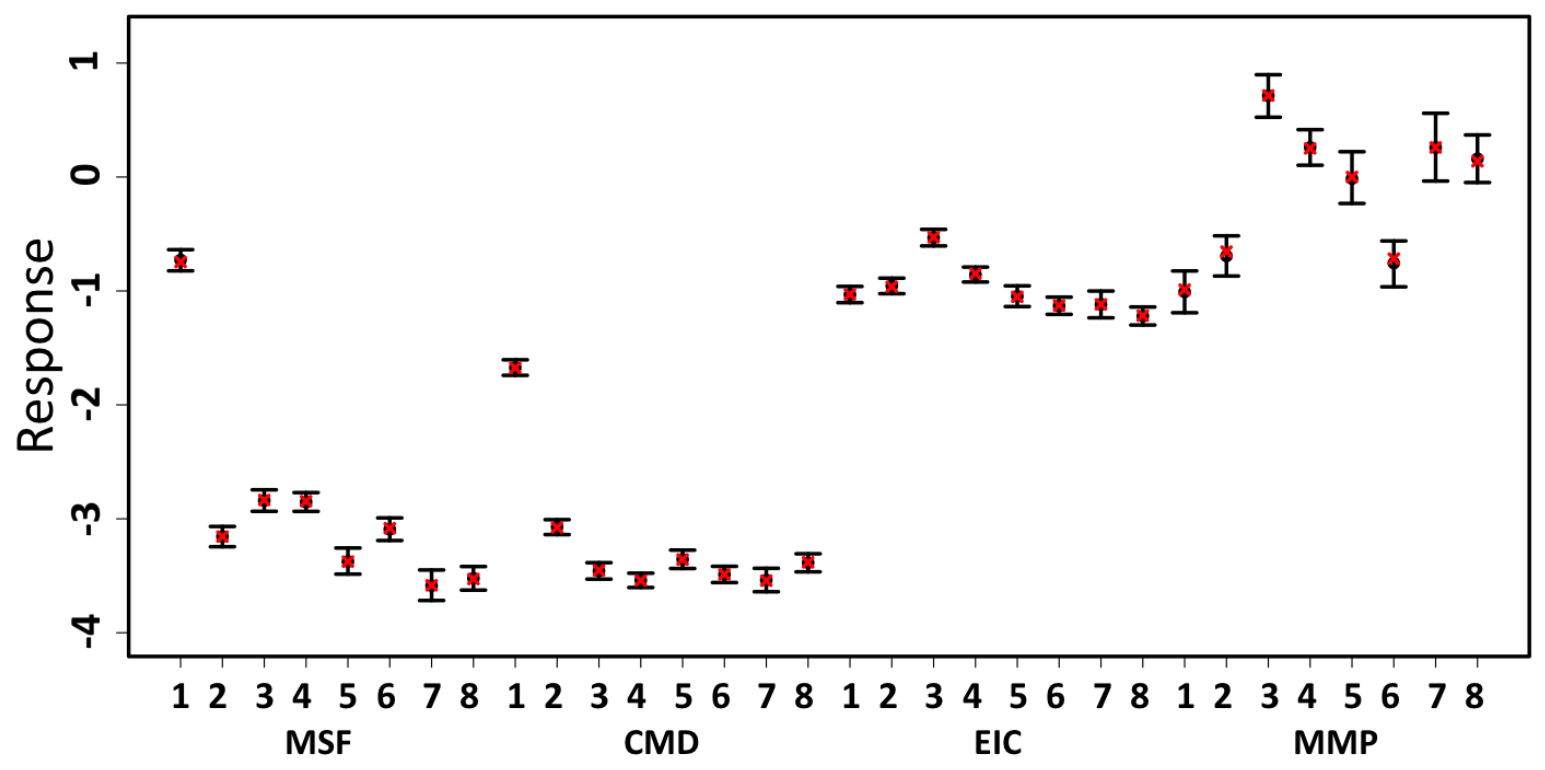

Figure 22: Posterior Predictive Check. Mean and 95\% posterior intervals of the posterior predictive mean response across all doses and times of exposure, for all outcomes and particles 1 through 8 (QD, $\mathrm{ZnO}, \mathrm{Fe}_{3} \mathrm{O}_{4}, \mathrm{Pt}, \mathrm{Ag}, \mathrm{SiO}_{2}, \mathrm{Al}_{2} \mathrm{O}_{3}, \mathrm{Au}$ ). Also included are the empirical mean responses across all doses and times of exposure (red). 